\title{
Specific covalent inhibition of MALT1 paracaspase suppresses B cell lymphoma growth
}

\author{
Lorena Fontán, ${ }^{1}$ Qi Qiao, ${ }^{2}$ John M. Hatcher, ${ }^{3,4}$ Gabriella Casalena, ${ }^{1}$ Ilkay Us, ${ }^{1}$ Matt Teater, ${ }^{1}$ Matt Durant, ${ }^{1}$ Cuangyan Du, ${ }^{3,4}$ Min Xia, ${ }^{1}$ \\ Natalia Bilchuk, ${ }^{1}$ Spandan Chennamadhavuni, ${ }^{3,4}$ Giuseppe Palladino, ${ }^{5}$ Giorgio Inghirami, ${ }^{5,6}$ Ulrike Philippar, ${ }^{7}$ Hao Wu, ${ }^{2}$ \\ David A. Scott, ${ }^{3,4}$ Nathanael S. Gray, ${ }^{3,4}$ and Ari Melnick ${ }^{1}$ \\ 'Division of Hematology and Oncology, Department of Medicine, Weill Cornell Medicine, Cornell University, New York, New York, USA. ²Program in Cellular and Molecular Medicine, Boston Children's Hospital, \\ Harvard Medical School, Boston, Massachusetts, USA; Department of Biological Chemistry and Molecular Pharmacology, Harvard Medical School, Boston, Massachusetts, USA. ${ }^{3}$ Department of Biological \\ Chemistry and Molecular Pharmacology, and ${ }^{4}$ Department of Cancer Biology, Dana-Farber Cancer Institute, Harvard Medical School, Boston, Massachusetts, USA. ${ }^{5}$ Department of Pathology and Laboratory \\ Medicine, Weill Cornell Medical College, New York, New York, USA. ${ }^{6}$ Department of Molecular Biotechnology and Health Sciences, University of Turin, Turin, Italy. ${ }^{7}$ Oncology Discovery, Janssen Research and \\ Development, Beerse, Belgium.
}

\begin{abstract}
The paracaspase MALT1 plays an essential role in activated B cell-like diffuse large B cell lymphoma (ABC DLBCL) downstream of $B$ cell and TLR pathway genes mutated in these tumors. Although MALT1 is considered a compelling therapeutic target, the development of tractable and specific MALT1 protease inhibitors has thus far been elusive. Here, we developed a target engagement assay that provides a quantitative readout for specific MALT1-inhibitory effects in living cells. This enabled a structure-guided medicinal chemistry effort culminating in the discovery of pharmacologically tractable, irreversible substrate-mimetic compounds that bind the MALT1 active site. We confirmed that MALT1 targeting with compound 3 is effective at suppressing ABC DLBCL cells in vitro and in vivo. We show that a reduction in serum IL-10 levels exquisitely correlates with the drug pharmacokinetics and degree of MALT1 inhibition in vitro and in vivo and could constitute a useful pharmacodynamic biomarker to evaluate these compounds in clinical trials. Compound 3 revealed insights into the biology of MALT1 in ABC DLBCL, such as the role of MALT1 in driving JAK/STAT signaling and suppressing the type I IFN response and MHC class II expression, suggesting that MALT1 inhibition could prime lymphomas for immune recognition by cytotoxic immune cells.
\end{abstract}

\section{Introduction}

Diffuse large B cell lymphoma (DLBCL) is the most common subtype of B cell non-Hodgkin lymphoma (1). Gene expression profiling enabled the subclassification of DLBCLs into distinct molecular subtypes, including germinal center B cell-like (GCB) DLBCL, activated $B$ cell-like $(A B C)$ DLBCL, and primary mediastinal B cell lymphoma $(2,3)$. These subtypes harbor diverse oncogenic lesions and are addicted to different signaling pathways, which render them sensitive or resistant to specific pathway inhibitors. Among the different DLBCL subtypes, ABC DLBCLs exhibit the most aggressive clinical behavior and are the least curable. Thus, the development of improved therapies for ABC DLBCL remains a high priority.

Constitutive activation of the NF- $\kappa \mathrm{B}$ signaling pathway is a molecular hallmark of ABC DLBCL and confers proliferation and survival advantages to these tumor cells $(2,4)$. This aberrant activation has been attributed to somatic mutations affecting protein components of the B cell receptor (BCR) as well as the TLR pathways (5-9). The most frequent activating mutations in the BCR pathway occur within the coding region of CD79A/B ( 20\% of ABC DLBCLs) (5) and CARD11/CARMA1 ( 10\%) (10), whereas

Conflict of interest: AM, NSG, and HW received funding from Janssen Pharmaceuticals. UP is an employee of Janssen Research and Development LLC.

Submitted: December 26, 2017; Accepted: July 9, 2018

Reference information: J Clin Invest. 2018;128(10):4397-4412.

https://doi.org/10.1172/JCI99436 the most frequently inactivated BCR-related tumor suppressor is A20 ( 23\%) (7, 8). Notably, 70\% of patients with ABC DLBCL show a gain or amplification of MALT1, which is located in 18q21 (11). In the TLR pathway, MYD88 is the most commonly mutated protein ( $37 \%$ of ABC DLBCL patients) (9).

As a part of the CARD11-BCL10-MALT1 (CBM) complex, MALT1 plays a pivotal role in B cell activation. MALT1 is a paracaspase that belongs to the caspase family of proteases and shows argininespecific cysteine protease activity $(12,13)$. Through proteolytic cleavage, MALT1 mediates the inactivation of inhibitors of the NF- $\mathrm{B}$ signaling pathway, such as TNFAIP3/A20 (12), and the activation of proteins that favor NF- $\mathrm{kB}$ activity, such as BCL10 (13), CYLD (14), RelB (15), and several others (16-19). In addition, MALT1 serves as a scaffold to recruit additional signal transducers to activate $\mathrm{NF}-\kappa \mathrm{B}$ signaling (20).

Previous studies showed that the protease activity of MALT1 is essential for the survival of ABC DLBCL cell lines that rely on constitutively active NF- $\kappa \mathrm{B}$ signaling, pointing to MALT1 as a compelling therapeutic target $(21,22)$. Along these lines, several small molecules and peptides that target MALT1 protease activity have been described but manifest significant liabilities, either because of poor selectivity or problematic pharmacologic properties (23-25). Moreover, it has been difficult to evaluate the molecular targeting of MALT1, given the lack of cell-based quantitative target engagement assays. Hence, the development of more viable and selective MALT1 inhibitors as 
A

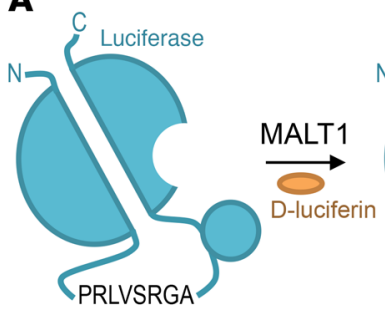

C

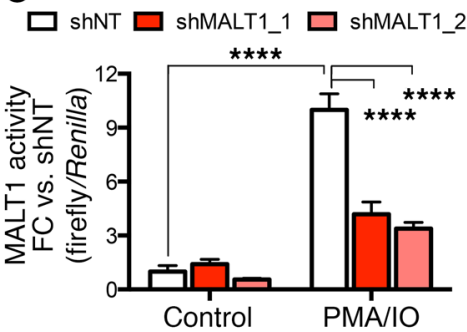

B

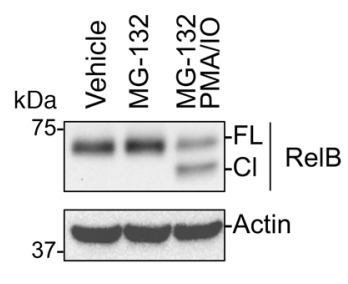

D

D

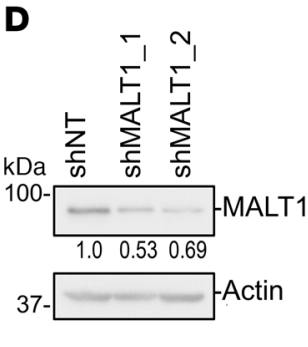

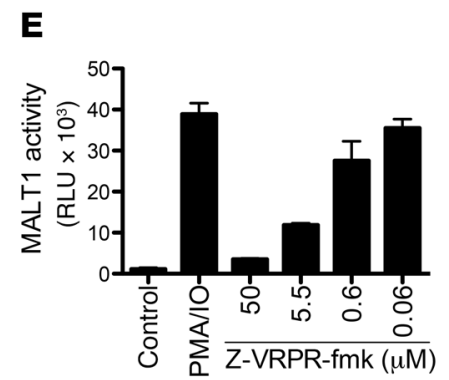

Figure 1. Development of a cell-based MALT1 protease reporter assay. (A) Schematic illustration of the MALT1-GloSensor reporter assay. C, carboxy terminus; N, amino terminus. (B) Induction of MALT1 protease activity in Raji cells treated with vehicle or $200 \mathrm{ng} / \mathrm{ml} \mathrm{PMA}$ and $1 \mu \mathrm{M} \mathrm{IO}$ for 1 hour. Protein was extracted and blotted for RelB. FL, full length; Cl, cleavage band. (C) Luciferase activity of the MALT1-GloSensor reporter was measured after MALT1 knockdown with 2 independent hairpins against MALT1 or a nontargeting (shNT) control and normalized to Renilla control. Cells were stimulated with vehicle or $200 \mathrm{ng} / \mathrm{ml}$ PMA and $1 \mu \mathrm{M} \mathrm{IO}$ for 2 hours. FC relative to the nontargeting shRNA (shNT). Results are representative of 2 independent experiments performed in triplicate. ${ }^{* * *} P<0.0001$, by ANOVA with Tukey's multiple comparisons adjustment. (D) MALT1 expression in MALT1-knockdown Raji MALT1-GloSensor reporter cells assayed in C. Numbers below the blot indicate MALT1 expression FC versus shNT (MALT1/actin). (E) Dose-dependent inhibition of MALT1 reporter activity in response to Z-VRPR-fmk. Cells were pretreated for 30 minutes with the inhibitor before PMA and IO stimulation, as in B. RLU, relative luciferase units. Data represent the mean \pm SD of 1 representative experiment.

well as useful pharmacodynamic (PD) assays is highly desirable to advance this field.

Most protease inhibitor drugs are active site inhibitors that are often developed using a substrate-based peptidomimetic approach. Several of these inhibitors, such as aliskiren (renin inhibitor) (26), ritonavir (HIV protease) (27), and carfilzomib (proteasome inhibitor) (28), have achieved a high degree of selectivity within the target class and reached the clinic. Toward the development of viable MALT1 inhibitors, we used a structure-function approach to design bona fide MALT1-selective, substrate-mimetic inhibitors and developed a suitable method to assess MALT1 target engagement.

\section{Results}

Development of a quantitative cell-based target engagement assay for MALT1 protease activity. High-throughput assessment of MALT1 inhibitor activity in living cells is limited by the lack of a suitable quantitative target engagement assay. We therefore developed a robust and sensitive method for the detection of intracellular MALT1 paracaspase activity. We used the GloSensor split luciferase method, which utilizes a bioluminescent chimeric protein composed of a genetically modified form of firefly luciferase split into 2 distinct domains by insertion of a cAMP-binding protein moiety (Figure 1A). Binding of cAMP induces a conformational change that reestablishes a functional luciferase protein resulting in luminescence. We engineered our reporter so that this conformational change, instead of being induced by cAMP, was caused by MALT1-induced cleavage, and hence luciferase activity would be a surrogate of endogenous protease activity. We used the RelB MALT1 cleavage site sequence in our GloSensor construct, so it would be specifically activated by MALT1 (Figure 1A). For the initial cell model, we used Raji lymphoma cells, which are easily transducible and suitable for such studies, since they activate MALT1 upon stimulation with PMA and ionomycin (IO) $(12,13)$. To further confirm the suitability of the RelB MALT1 cleavage site as a substrate, we treated Raji cells with PMA and IO for 1 hour and analyzed RelB cleavage by Western blotting. Exposure of Raji cells to PMA and IO induced MALT1 protease activity as detected by the presence of the RelB cleaved band (Figure 1B).

Next, we generated a stable Raji MALT1-GloSensor reporter cell line and observed that luciferase activity was induced 10-fold following PMA and IO treatment (Figure 1C) (ANOVA followed by Tukey's multiple comparisons test; $P<0.0001)$. To verify MALT1 specificity, Raji cells expressing the MALT1-GloSensor reporter were infected by lentiviruses expressing either MALT1 shRNAs or a nontargeting control (shNT). We found that MALT1 knockdown caused a significant reduction in luciferase activity (by $58 \%$ and $66 \%$ for shMALT1_1 and shMALT_2; ANOVA followed by Tukey's multiple comparisons test; $P<0.0001$ for both shRNAs), which was proportional to the knockdown efficiency of the shRNAs (Figure 1D), demonstrating that the GloSensor reporter activity was MALT1 specific. As an additional control, we tested whether the specific and irreversible MALT1 inhibitor peptide Z-VRPRfmk could extinguish GloSensor activation by PMA and IO. Raji MALT1-GloSensor cells were pretreated with various doses of Z-VRPR-fmk for 30 minutes and then induced with PMA and IO for 1 hour. We observed that increasing concentrations of Z-VRPR-fmk resulted in a dose-dependent decrease in luciferase activity (Figure 1E). To rule out artifact due to interference of pep- 
A

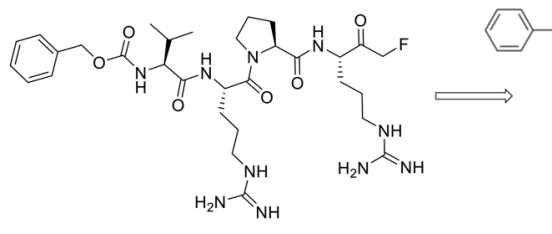

Z-VRPR-fmk

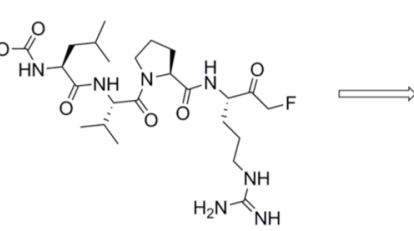

Compound 1

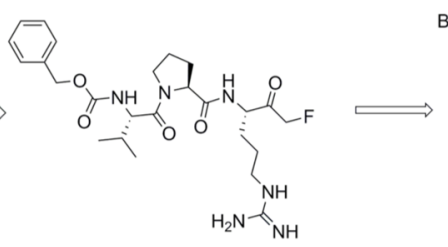

Compound 2

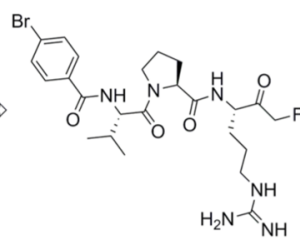

Compound 3
B

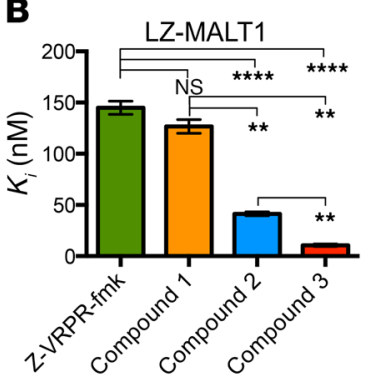

C

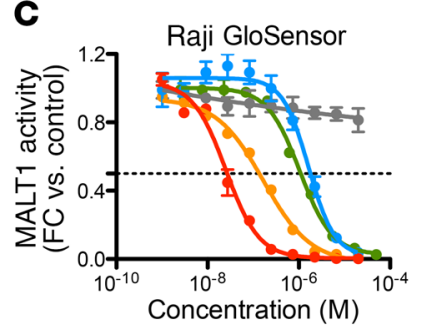

D

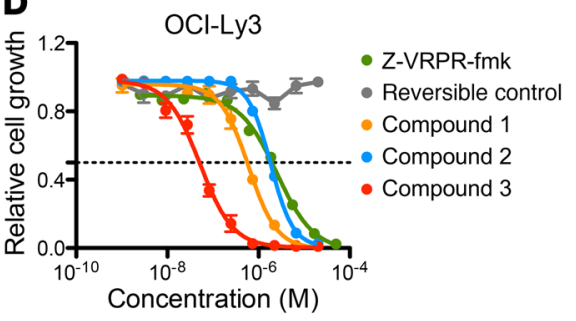

Figure 2. Compound 3 shows maximal inhibition of MALT1 activity in in vitro and cell-based assays. (A) Schematic illustration of the chemical structures of MALT1 inhibitors based on Z-VRPR-fmk. (B) In vitro MALT1 $K_{i}$ values for the examples in A in an LZ-MALT1 biochemical assay. Results represent the mean \pm SEM of 3 independent experiments. ${ }^{* *} P<0.01$ and ${ }^{* * *} P<0.0001$, by ANOVA with Tukey's multiple comparisons adjustment. (C) Cell-based reporter assay showing inhibition of cellular MALT1 activity in Raji MALT1-GloSensor cells following treatment with different inhibitors, as in Figure 1. Dashed line indicates $\mathrm{CI}_{50}$. Results represent the mean \pm SEM of at least 3 independent experiments. (D) Growth suppression in the MALT1-sensitive cell line $\mathrm{OCI}-\mathrm{Ly} 3$ in response to the indicated MALT1 inhibitors. Graph shows cell growth relative to vehicle-treated cells and the concentration of the compound. Dashed line indicates $\mathrm{GI}_{50}$. Results are the average \pm SEM of at least 3 independent experiments.

tides with the luminescence signal, we tested Renilla activity in parallel, which indeed was not affected by Z-VRPR-fmk (Supplemental Figure 1; supplemental material available online with this article; https://doi.org/10.1172/JCI99436DS1).

Development of a selective substrate-mimetic inhibitor of MALT1. In order to develop superior MALT1 catalytic activity inhibitors, we used the following 3 different assays to support structureactivity relationship (SAR) studies: (a) an in vitro biochemical assay using a recombinant form of MALT1 (aa 340-789) fused to a leucine zipper dimerization motif (LZ-MALT1) that promotes MALT1 dimerization and activation (23); (b) an assay using the above-described cell-based GloSensor reporter that measures MALT1 protease activity in live cells (Figure 1, A-E); and (c) a differential growth inhibition assay of ABC versus GCB DLBCL cell lines. Equipped with these tools, we used Z-VRPR-fmk as a starting point to develop substrate-mimetic MALT1 inhibitors.

Z-VRPR-fmk was derived from the optimal tetrapeptide substrate for the metacaspase AtmC9 (29) and incorporates an electrophilic fluoromethyl ketone warhead, which forms a covalent bond with the active site cysteine residue (Figure 2A). Although Z-VRPR-fmk has detectable activity in cell-based assays $(22,30)$, its efficacy is highly limited because of poor cell penetration, probably due to the 2 arginine residues. Previous studies of MALT1 substrate specificity based on positional scanning libraries (31, 32 ) and co-crystal structures with Z-VRPR-fmk $(31,33)$ had suggested that the $\mathrm{P} 1$ arginine might be critical, given the multiple interactions with acidic residues in the $\mathrm{P} 1$ pocket, but that the $\mathrm{P} 3$ arginine could be replaced (Supplemental Figure 2A). Moreover, of 11 reported MALT1 substrates, only BCL10 has a P3 arginine, whereas all of them have a P1 arginine (34). An activity-based probe for MALT1 using the LVSR tetrapeptide sequence was also confirmed to bind MALT1 specifically (35). Therefore, we replaced arginine with valine at $\mathrm{P}$, and, in order to minimize the number of $\mathrm{H}$-bond donors, we chose to keep proline at $\mathrm{P} 2$ and generated Z-LVPR-fmk (compound 1) (Figure 2A). Compound 1 had a $K_{i}$ similar to that of Z-VRPR-fmk in the biochemical assay (Figure 2B) but displayed a 10-fold improvement in cell-based, Raji MALT1-GloSensor protease inhibition (Figure 2C), and OCI-Ly3 growth inhibition (Figure 2D) assays, probably due to improved cell permeability. The reversible analog of compound 1 (reversible control; Supplemental Figure 2B) that is based on the same tetrapeptide but lacks the fmk group showed no effects in the cellular MALT1-GloSensor protease inhibition or cell proliferation assays (Figure 2, C and D), in agreement with the finding of no activity against LZ-MALT1 at $5 \mu \mathrm{M}$ (LZ-MALT1 inhibition $<2 \%$ ). These data confirm the importance of covalent binding to the MALT1 catalytic site in achieving potent inhibition of MALT1 protease activity.

Next, to allow for easier preparation of compounds with potentially improved pharmacological properties, we also evaluated covalent tripeptides. A previous study using the Ac-VSRACC substrate showed that MALT1 was unable to cleave substrates shorter than tetrapeptides (32). While we found that a VPR-fmk tripeptide with a methylamine cap at the N-terminus showed very little activity in our assays, in contrast, a VPR-fmk tripeptide with a Cbz protecting group (compound 2, Z-VPRfmk) lost some cellular potency while retaining good in vitro potency compared with Z-VRPR-fmk (Figure 2, A-D). This made it a more attractive starting point for further optimization, given the ease of synthesis and reduced molecular weight. Therefore, it became the lead for further optimization of the series that ultimately yielded the 4-bromo-benzamide derivative compound 3. This compound exhibited 10- and 100-fold improved potency 
in vitro and in vivo, respectively, compared with Z-VRPR-fmk (Figure 2, A-D). Compound 3 was also significantly (3-fold) more potent in vitro than compound 2 (Figure $2 \mathrm{~B}$ ), while it was 35 -fold and 73-fold more potent in the Raji MALT1-GloSensor protease inhibition and OCI-Ly3 growth inhibition assays, respectively (Figure 2, C and D, and Supplemental Table 1). All the compounds retained excellent ABC versus GCB DLBCL differential growth inhibition (Supplemental Table 1). During our efforts to develop early peptidomimetic MALT1 inhibitors, we studied $\mathrm{IC}_{50}$ values of 111 derivatives of Z-VRPR-fmk in the MALT1GloSensor and OCI-Ly3 growth inhibition $\left(\mathrm{GI}_{50}\right)$ assays. $\mathrm{IC}_{50}$ and $\mathrm{GI}_{50}$ values for those assays in our SAR series showed a very significant correlation (Pearson's $R=0.78, P<0.001$ ) for all the Z-VRPR-fmk-derivative peptides (Supplemental Figure 2C). This is an indicator of the robustness of the GloSensor approach for the study of endogenous MALT1 protease activity and the selectivity of the compound series for its target in cells.

To more precisely assess the specificity of compound 3 for MALT1, we tested its activity against a panel of 26 cysteine proteases including caspases, cathepsins, and others (Supplemental Table 2). We found that compound 3 had minimal activity against these other proteases and inhibited only 3 of them by more than $50 \%$ at $100 \mathrm{nM}$, which was 10 -fold weaker than its effect on MALT1 in vitro. Therefore, our rationally designed MALT1 active site protease inhibitor, compound 3, has excellent biochemical and cellular potency and a good overall protease selectivity profile.

Structural basis for improved compound 3 activity involves aberrant MALT1 oligomerization. To further define the structural and biochemical features of peptidomimetic MALT1 inhibitors, we used compound 2 or compound 3 to form complexes in vitro with the purified MALT1 caspase-Ig3 domain. While it is known that MALT1 alone forms dimers upon activation or by binding to substrate-mimetic ligands (31) in the context of the CBM complex, MALT1 activation may rely on induced proximity within the higher-order structure assembly of BCL10 filaments (36). Therefore, aiming to determine the impact of our compounds on MALT1 assembly, we subjected MALT1 compound complexes to gel filtration and multi-angle light scattering (MALS), which is used to calculate the molecular mass of oligomeric assemblies. We observed that compound 2 induced MALT1 dimerization, similar to what we observed and was also reported for Z-VRPR-fmk (Figure 3A) (31). In contrast, the compound 3-MALT1 complex formed larger oligomers, peaking at a molecular mass of approximately $450 \mathrm{kDa}$ (4\% error), in addition to smaller peaks of dimers and monomers (monomer molecular weight $[\mathrm{MW}]=44 \mathrm{kDa}$ ) (Figure $3 \mathrm{~A}$ and Supplemental Figure 3A). Chymotrypsin digestion of the large oligomer fraction resulted in the removal of the Ig3 domain and reduced the oligomer mainly to a compound 3-MALT1caspase domain tetramer with a molecular mass of approximately $102 \mathrm{kDa}$ (5\% error) and a dimer with a molecular mass of approximately $58 \mathrm{kDa}$ ( $4 \%$ error) by MALS (monomer MW=27 kDa) (Figure $3 \mathrm{~B}$ and Supplemental Figure 3, B and C), indicating that both domains, caspase and Ig3, contributed to oligomerization.

To elucidate the mechanism of action of these drugs, we solved the crystal structures of the compound 2-MALT1 dimer and the compound 3-MALT1-caspase domain tetramer at $2.2 \AA$ and $1.9 \AA$ resolution, respectively (Supplemental Table 3). The electron density clearly revealed the covalent bond between the active site residue C464 and the ligands (Figure 3C). Compared with Z-VRPRfmk, the tripeptides maintained the main chain interactions with MALT1, but differed in capping group orientations (Figure 3D). The Cbz group of compound 2 occupied a hydrophobic pocket for the valine in Z-VRPR-fmk and stacked with F499 (Figure 3D), which increased the binding affinity and explained its improved inhibition of MALT1 in vitro. In contrast, the 4-Br-phenyl capping group of compound 3 could not associate with the hydrophobic pocket because of the shortened linker and instead interacted with a Y389 residue from a neighboring MALT1-caspase domain dimer (Figure 3, C and E), which is consistent with the tetramer observation in solution. Compared with the dimer orientation in the MALT1-VRPR complex structure, one MALT1-compound 3 subunit slides $4.5 \AA$ in the dimer structure, which contributes to the formation of the tetramer interface (Figure 3, E and F). Residue Y389 shifts 6.6 A from its position in the MALT1-VRPR structure and forms a halogen bond with the 4-Br in compound 3 (Figure 3G). In addition, residues E388, E396, K404, E437, H540, and $\mathrm{K} 543$ locate in an interface between caspase domain dimers, which may help stabilize the compound 3-MALT1 oligomer (Figure $3 \mathrm{E})$. Notably, removal of the 4-Br from compound 3 abolished MALT1 oligomerization (Figure $3 \mathrm{H}$ ) and significantly reduced its potency in vitro and in vivo $\left(K_{i}=10 \mathrm{nM}\right.$ vs. $71 \mathrm{nM}$, GloSensor IC $_{50} 0.06 \mu \mathrm{M}$ vs. $0.65 \mu \mathrm{M}$, OCI-Ly3 $\mathrm{GI}_{50} 0.10 \mu \mathrm{M}$ vs. $0.66 \mu \mathrm{M}$ ), suggesting that compound 3-induced oligomerization may contribute to MALT1 inhibition.

Response to compound 3 precisely defines the degree of MALT1 dependency in $A B C D L B C L s$. We next examined the effects of compound 3 on cell growth using a large panel of ABC DLBCL and GCB DLBCL lymphoma cell lines. We found that compound 3 significantly suppressed the growth of the ABC DLBCL cell lines HBL1, TMD8, OC1-Ly10, and OCI-Ly3 compared with that of all other cell lines $(P<0.0001$, ANOVA) (Figure $4 \mathrm{~A}$ and Supplemental Table 4), suggesting that these cells are MALT1 dependent. In contrast, other ABC DLBCLs (U2932, SU-DHL-2, HLY1, RC-K8) and all of the GCB DLBCLs (OCI-Ly7, OCI-Ly1, SU-DHL-4) were resistant to compound 3 (Figure $4 \mathrm{~A}$ ), suggesting that these cell lines do not rely on MALT1 protease activity for their growth. Even at a concentration of $20 \mu \mathrm{M}$, nearly 200 -fold higher than the $\mathrm{GI}_{50}$ of the sensitive cell lines, compound 3 inhibited cell growth by less than $15 \%$ in the resistant cell lines (Supplemental Table 4).

To further explore the basis for MALT1 paracaspase dependency of particular DLBCL cell lines, we assessed MALT1 activity by monitoring the cleavage status of RelB. Cell lines were pretreated with vehicle or $200 \mathrm{nM}$ compound 3 for 30 minutes, followed by an additional 1.5 hours of treatment with MG-132 (to block degradation of the cleavage products) and then analysis by Western blotting using an antibody directed against RelB. As shown in Figure 4B, all MALT1-dependent cell lines manifested MALT1 paracaspase activity, as evidenced by the presence of RelB cleavage products after MG-132 treatment. In contrast, MALT1 was not active in MALT1-independent cell lines, except for U2932 (Figure 4B). Notably, administration of compound 3 caused efficient inhibition of RelB cleavage in MALT1dependent cells as well as U2932 cells, whereas all cell lines neg- 
A

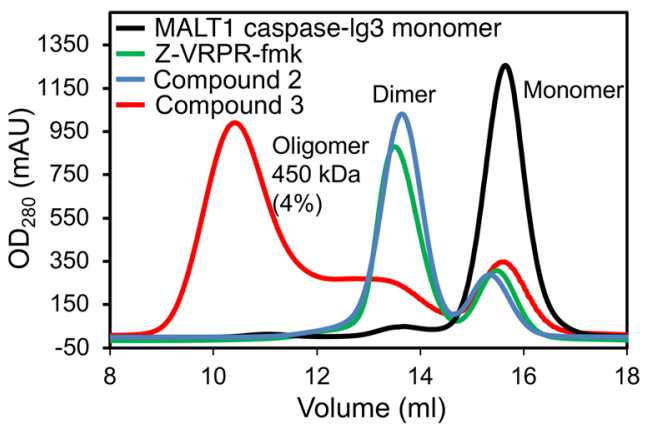

C

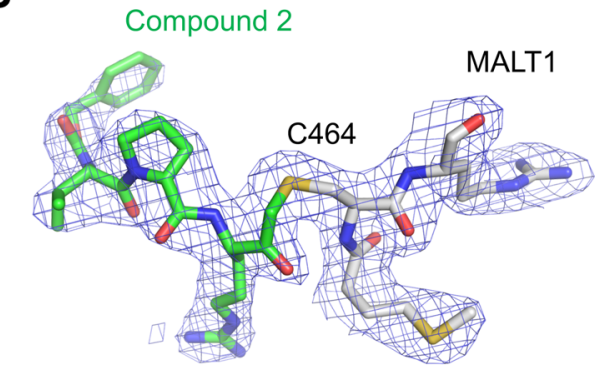

E

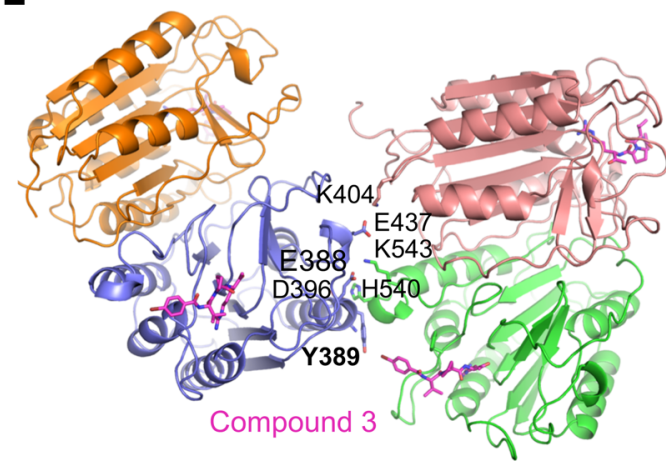

G

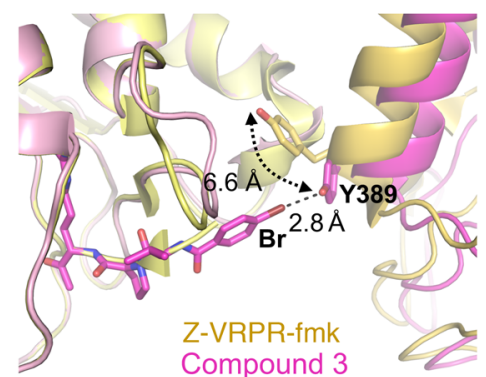

B

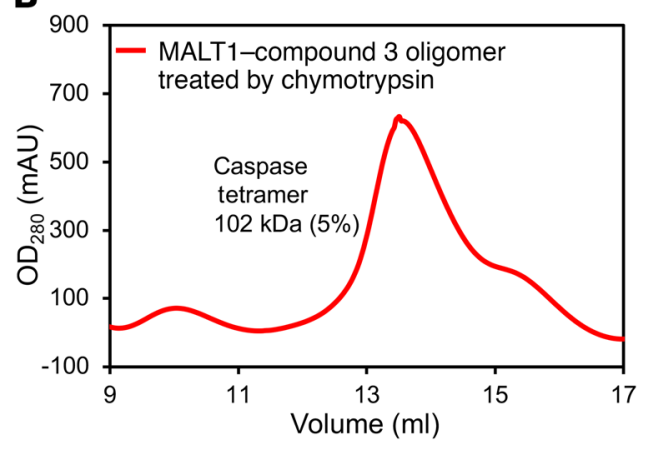

D

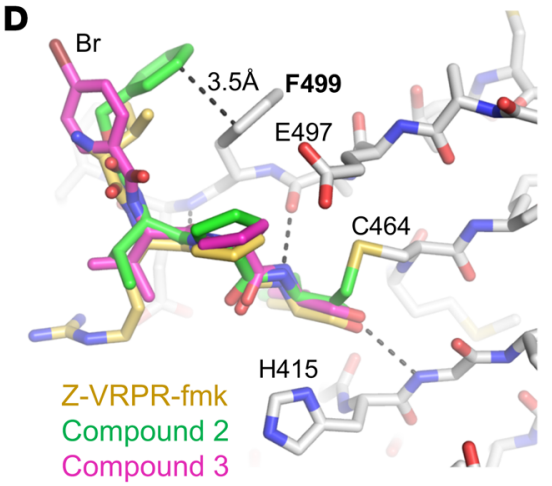

$\mathbf{F}$

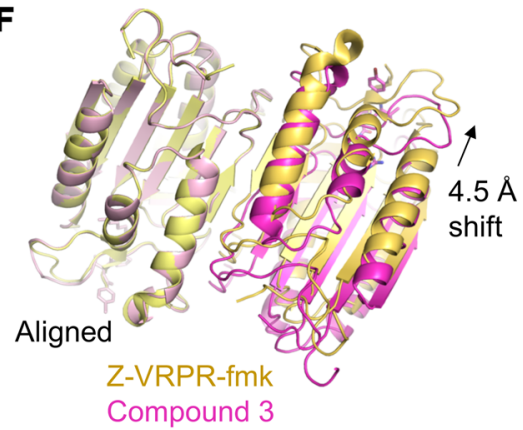

H

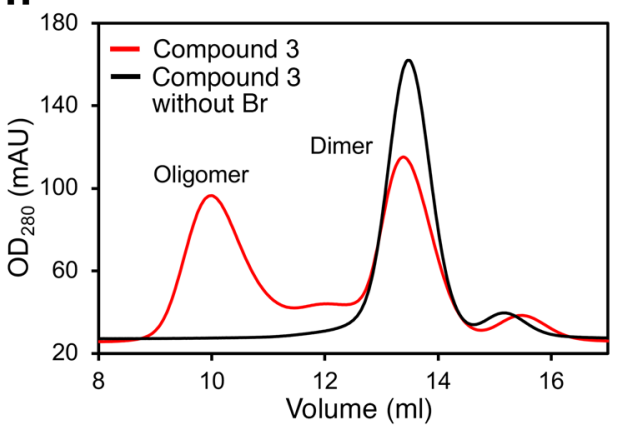

Figure 3. Compound 3 binds covalently to the catalytic cysteine of MALT1. (A) MALS with in-line gel filtration chromatography showing that compound 3 induced MALT1 oligomerization, while other inhibitors predominantly induced dimerization. The measured molecular masses by MALS are shown. The molecular mass measurement error is shown in parentheses. (B) Molecular mass measured by MALS showing that after chymotrypsin treatment, which removes the Ig3 domain, compound 3 mainly induced caspase domain tetramerization. The molecular mass measurement error is shown in parentheses. (C) The $2 \mathrm{Fo}-\mathrm{Fc}$ map $(1.0 \sigma)$ of the MALT1compound 2 complex crystal structure shows the covalent bond between inhibitor and MALT1 active site residue C464. (D) Structure alignment showing that the binding of MALT1 inhibitors mainly differs in the capping group orientation. (E) Compound 3-MALT1 tetramer structure showing the interface between dimers. (F) Structure alignment between compound 3-MALT1 caspase and VRPR-MALT1 caspase dimers showing differences in dimer orientation. (C) The 4-Br group of compound 3 forms a halogen bond with Y389 of a neighboring MALT1 molecule. (H) Gel filtration analysis of compound 3-MALT1 caspase compared with the same compound without $\mathrm{Br}$, suggesting that $4-\mathrm{Br}$ is necessary for compound 3-induced MALT1 oligomerization. ative for MALT1 activity were resistant to compound 3. U2932 was previously reported to harbor a S417A mutation in TGF- $\beta$ activated kinase 1 (TAK1), which activates $\mathrm{NF}-\kappa \mathrm{B}$ signaling downstream of MALT1 in the BCR pathway (8). Although the significance of TAK1 S417A mutation has not been established, U2932 cells were sensitive to the TAK1 inhibitor (5Z)-7oxozeaenol (Supplemental Figure 4A). Therefore, TAK1 mutations should be considered before treatment with MALT1 inhibitors or other inhibitors upstream in the pathway, such as ibrutinib. Taken together, these data demonstrate that compound 3 is a highly selective inhibitor with virtually no toxic effects in MALT1-independent cell lines. Another feature of most MALT1 inhibition-resistant ABC DLBCL cell lines (3 of 4) is TNFAIP3 loss. Homozygotic loss of TNFAIP3 in ABC DLBCL (RC-K8, SU-DHL-2, and HLY1) correlates with a lack of MALT1 activity and, consequently, a lack of response to MALT1 inhibition. We do not understand how TNFAIP3 loss results in attenuated MALT1 activity. However, TNFAIP3 lesions have 
A

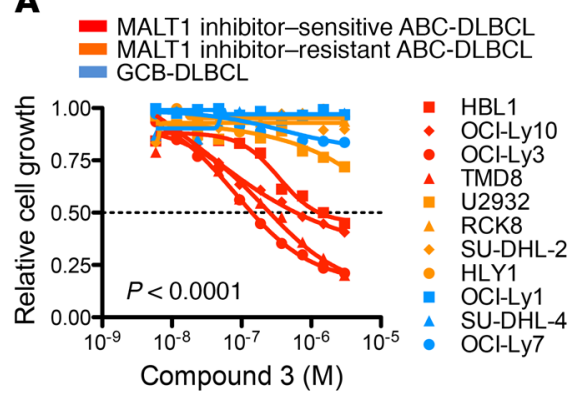

C

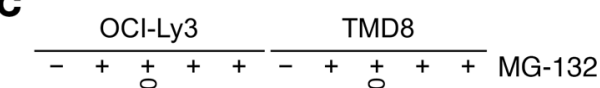

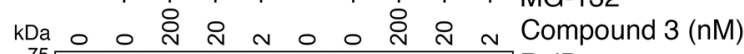

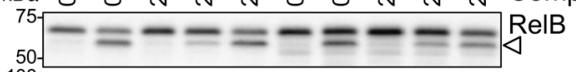

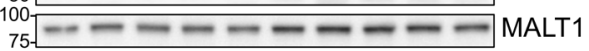

37 - - - - - Actin

B

B ABC-DLBCL (MALT1 inhibitor-sensitive)

ABC-DLBCL (MALT1 inhibitor-resistant)

GCB-DLBCL (MALT1 inhibitor-resistant)

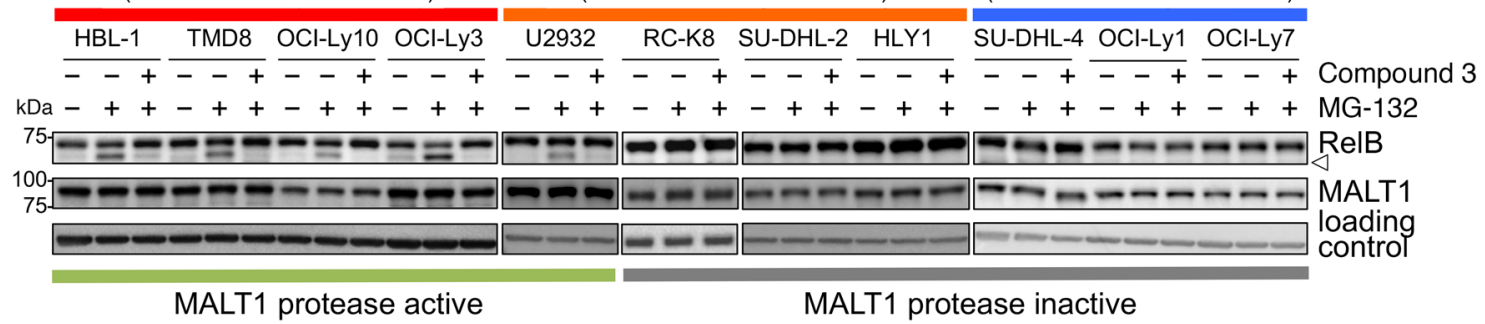

D
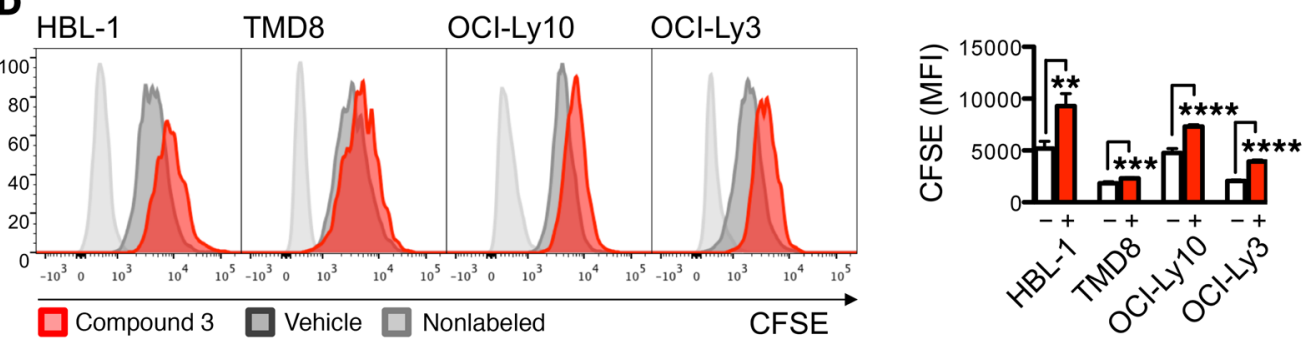

Compound 3

E

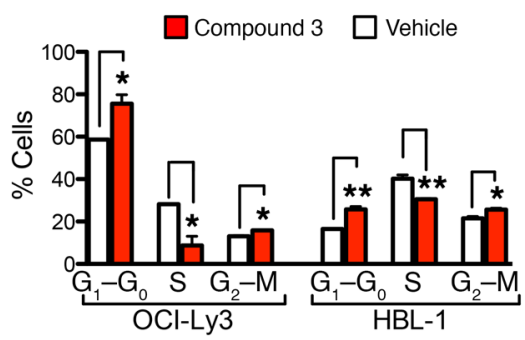

$\mathbf{F}$

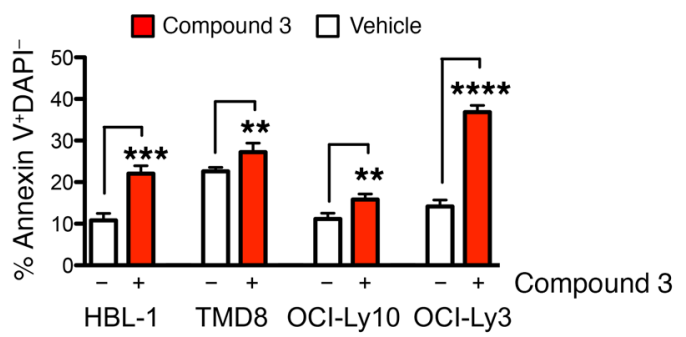

Figure 4. Compound 3 selectively inhibits the growth of MALT1-dependent cell lines by decreasing cell proliferation and increasing apoptosis. (A) Compound 3 effect on cell growth over DMSO control in a panel of 11 cell lines. Cells were exposed to compound for 4 days. Results represent the average of 2 independent experiments. (B) Western blot analyses of RelB and MALT1 after a 30-minute pretreatment with 200 nM compound 3 or vehicle, followed by $5 \mu \mathrm{M}$ MG-132 proteasome inhibitor for 1.5 hours in the indicated cell lines. Arrowhead indicates the cleaved product. (C) Dose-dependent RelB cleavage inhibition by compound 3. Cells were treated as in B with the indicated amounts of compound 3. (D) Assessment of proliferation by CFSE dilution in the indicated cell lines after treatment with $1 \mu \mathrm{M}$ compound 3 for 6 days. DAPI' single cells ( $10^{4}$ cells) were gated for analysis. Bar graph shows CFSE mean fluorescence intensity (MFI). Data represent the mean \pm SD of 1 representative experiment performed in triplicate. The experiment was performed 3 times with similar results. (E) OCI-Ly3 and HBL-1 cells treated with $1 \mu \mathrm{M}$ compound 3 for 24 hours were BrdU pulse labeled for 15 minutes, and cell-cycle distribution was analyzed by flow cytometry. Bar graph shows the percentage of cells in different phases of the cell cycle following treatment with vehicle or compound 3. Data represent the mean \pm SD of 2 independent experiments. (F) Apoptosis was assessed by annexin $\mathrm{V}^{+} \mathrm{DAPI} \mathrm{I}^{-}$in cell lines treated with compound 3 for 6 days (treatment was repeated every $48 \mathrm{~h}$ ). The $y$ axis shows the percentage of annexin $\mathrm{V}^{+} \mathrm{DAPl}{ }^{-}$cells. Data represent the mean \pm SD of 1 representative experiment. Each experiment was performed at least twice with similar results. ${ }^{*} P<0.05,{ }^{* *} P<0.01,{ }^{* *} P<0.001$, and ${ }^{* * * *} P<0.0001$, by unpaired, 2-tailed Student's $t$ test.

been shown to confer ibrutinib resistance in $A B C$ DLBCL patients (37) and cell lines (38).

To determine whether the observed biological effects of compound 3 treatment were linked to its effects on MALT1 paracaspase activity, we monitored the impact of increasing concentrations (2, 20, and $200 \mathrm{nM})$ of compound 3 on RelB cleavage inhibition in MALT1-dependent DLBCL cells. As shown in Figure 4C and Supplemental Figure 4B, proteolysis of RelB was impaired in a dose-dependent manner. At $200 \mathrm{nM}$ compound 3, RelB cleavage was inhibited by $96 \%$ in OCI-Ly3, 
A

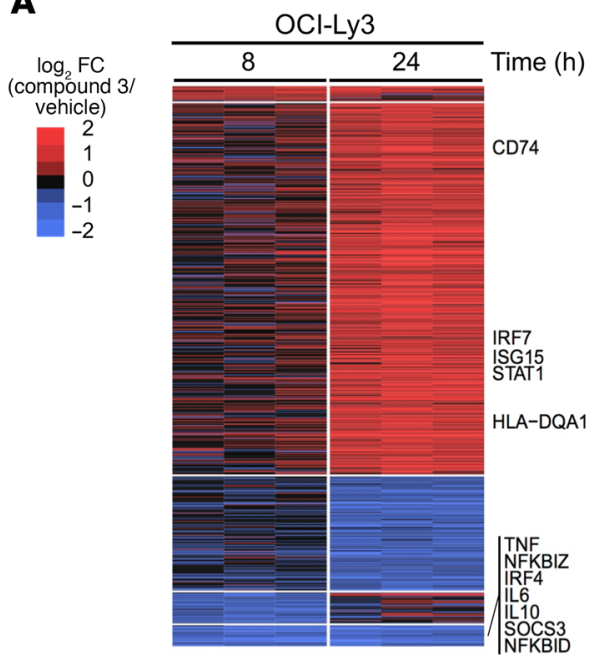

B
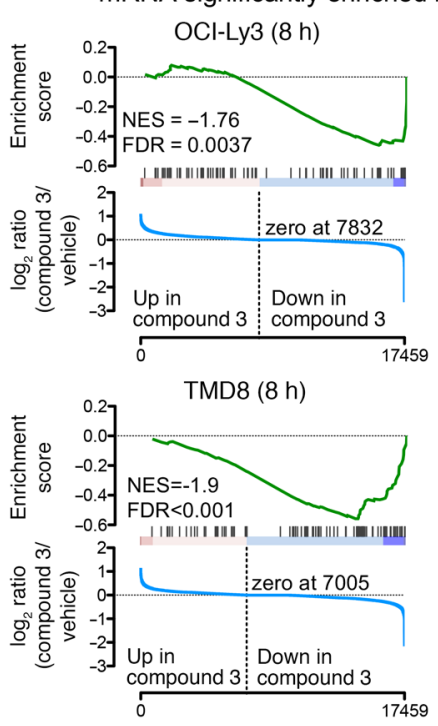

D
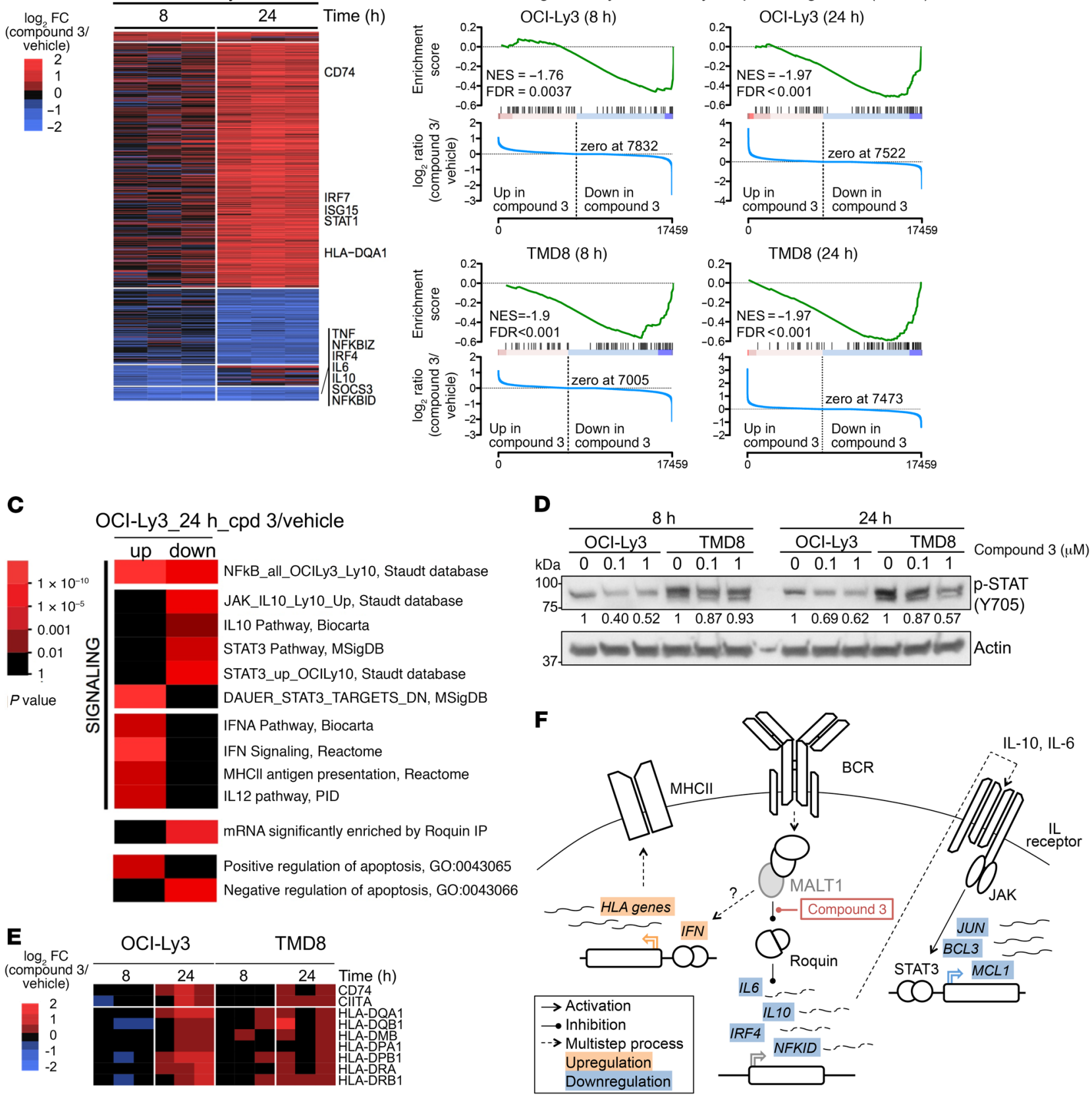

Figure 5. Compound 3 regulates immune signaling. (A) Heatmap showing differentially expressed genes in OCI-Ly3 cells treated for 8 or 24 hours with compound 3 relative to their vehicle controls. Highlighted are genes for relevant significantly enriched pathways: Roquin targets, JAK/STAT pathway, or MHC class II [MHCII]) genes. (B) GSEA for Roquin targets in OCI-Ly3 and TMD8 at 8 and 24 hours. cpd, compound; veh, vehicle. (C) Heatmap showing statistically significant pathways enriched in the upregulated or downregulated genes 24 hours after compound 3 treatment in OCI-Ly 3 cells by hypergeometric test. PID, Pathway Interaction Database. (D) Western blot for p-STAT3 (Y705) in cell extracts of OCI-Ly3 and TMD8 treated for 8 or 24 hours with 100 nM or $1 \mu \mathrm{M}$ compound 3. Results are representative of 2 experiments. (E) Heatmap of MHC class II genes showing log 2 FC expression of compound 3-treated cells versus vehicle-treated cells for OCI-Ly3 and TMD8 at 8 and 24 hours. (F) Schematic representation of the changes in mRNA expression for compound 3-regulated genes and their relationship to MALT1.

93\% in OCI-Ly10, $88 \%$ in TMD8, and 30\% in HBL-1 cells. The level of MALT1 inhibition by compound 3 was congruent with the growth-suppressive effects observed in the various cell lines. In fact, we observed a significant negative correlation (Pearson's $R=-0.97, P=0.02$ ) between MALT1 inhibition (calculated as the percentage of RelB cleavage inhibition at 200
nM compound 3) and compound $3 \mathrm{GI}_{50}$ in MALT1-dependent cell lines (Supplemental Figure 4C).

Specific inhibition of MALT1 paracaspase suppresses the proliferation and survival of DLBCL cells. To further characterize the cellgrowth inhibition mechanism of compound 3 , we investigated its effects on proliferation and apoptosis. ABC DLBCL cell lines (HBL- 
1, TMD8, OCI-Ly10, and OCI-Ly3) were labeled with CFSE, and cell division was tracked after compound 3 treatment by flow cytometric analysis of dye dilution in viable cells. As shown in Figure 4D, CFSE dilution was markedly delayed in compound 3-treated cells compared with vehicle-treated cells, demonstrating a significant decrease in cell proliferation (Figure 4D). To determine whether compound 3-mediated growth suppression is linked to cell-cycle arrest, we quantified the cell-cycle distribution of OCI-Ly3 and HBL-1 cells by BrdU pulse labeling. After a 24-hour exposure $(1 \mu \mathrm{M}$ compound 3), we observed a significant accumulation of cells in $G_{0}-$ $\mathrm{G}_{1}$ phases $(\triangle 29 \%$ and $56 \%$ for OCI-Ly3 and HBL-1, $P=004$ and $P$ $=0.002$, 2-tailed Student's $t$ test, respectively) that was associated with a significant decrease in S-phase entry $(\Delta-69 \%$ and $-24 \%$ for OCI-Ly3 and HBL-1, $P=0.05$ and $P=0.005$, 2-tailed Student's $t$ test, respectively) (Figure $4 \mathrm{E}$ ). To assess the effects of compound 3 on apoptosis, HBL-1, TMD8, OCI-Ly10 and OCI-Ly3 cell lines were exposed to $1 \mu \mathrm{M}$ concentration compound 3 for 6 days, and apoptosis was analyzed by annexin V and DAPI flow cytometry. We found that compound 3 significantly increased the fraction of annexin $\mathrm{V}^{+}$ cells (Figure 4F and Supplemental Figure 4D), indicating that compound 3 induced apoptosis in ABC DLBCL cells.

MALT1 inhibition extensively reprograms immune signaling pathways in $A B C D L B C L$ cells. The more potent and specific activity of compound 3 as compared with previous MALT1 inhibitors afforded us the opportunity to more fully explore pathways downstream of MALT1 paracaspase activity. We therefore performed RNAsequencing (RNA-seq) in OCI-Ly3 cells, which was the cell line most sensitive to MALT1 inhibition. We treated cells for 8 and 24 hours with $100 \mathrm{nM}$ compound 3 to study early and delayed effects of MALT1 paracaspase inhibition at the mRNA level. After 8 hours, compound 3 treatment mainly resulted in downregulation of gene expression, with 71 genes downregulated and 19 genes upregulated (fold change $[\mathrm{FC}]>1.2$ and adjusted $P<0.01$ ) (Figure $5 \mathrm{~A}$ ). Given the early nature of these changes in gene expression, such downregulation is probably a direct effect stemming from loss of MALT1 activity. After 24 hours, we observed secondary waves of transcriptional changes in OCI-Ly3, which now showed a shift toward gene upregulation, with upregulation of 533 genes versus downregulation of 189 genes $(\mathrm{FC}>1.2$ and adjusted $P<0.01)$. To confirm these findings, we treated TMD8 cells with $100 \mathrm{nM}$ compound 3 for 8 or 24 hours and performed RNA-seq. As with OCI-Ly3, we observed a wave of downregulated genes at 8 hours in TMD 8 cells (with downregulation of 140 genes and upregulation of 5 genes, $\mathrm{FC}>1.2$ and adjusted $P<0.01$ ), while at 24 hours, there were considerably fewer differentially expressed genes in TMD8 cells than in OCI-Ly3 cells (30 genes were upregulated and 52 were downregulated, FC $=1.2$ and adjusted $P<0.01)$. Gene set enrichment analysis (GSEA) of these differentially expressed genes used as signatures showed significant enrichment of the OCI-Ly3 signatures in TMD8 cells and vice versa (Supplemental Figure 5A). The more extensive gene expression changes in OCI-Ly3 cells were concordant with the fact that this is the cell line most sensitive to MALT1 inhibition $\left(\mathrm{GI}_{50}=87 \pm 6 \mathrm{nM}\right)$, as shown in Figure 4 and Supplemental Table 4. As expected, Z-VRPR-fmk signatures obtained in HBL-1 and TMD8 cells using microarrays were significantly enriched in compound 3-treated OCI-Ly3 and TMD8 cells at 24 hours (Supplemental Figure 5B).
Remarkably, $50 \%$ of the overlapping downregulated genes in OCI-Ly3 and TMD8 cells 8 hours after compound 3 treatment were Roquin targets (Supplemental Figure 5C). Roquin is a nuclease that degrades mRNAs related to inflammation and immunity including, among others, NFKBID, IL1O, and IL6 (17). Roquin is inactivated by MALT1-mediated proteolytic cleavage (17, 39), therefore, MALT1 inactivation is linked to reduced expression of Roquin targets. Consistent with this finding, we detected significant enrichment of a "Roquin targets" signature (40) among genes downregulated by compound 3 in both cell lines and at both time points using GSEA (Figure 5B) or a hypergeometric test (Roquin targets, $P=7.60 \times 10^{-6}$ ) (Figure $5 \mathrm{C}$ ). Compound 3-mediated downregulation of the Roquin targets NFKBID, IL1O, IL6, and RC3H1 was confirmed by quantitative PCR (qPCR) in both OCI-Ly3 and TMD8 cells (Supplemental Figure 5D), while TNF was significantly downregulated only in OCI-Ly3 at 8 hours.

Pathway analysis using a hypergeometric test in OCI-Ly3 compound-treated cells showed that IL-10 target genes and STAT3 signatures were significantly downregulated (STAT3 pathway genes, MSigDB [STKE], $P=8.3 \times 10^{-5}$ and STAT3_up_ OCILy10, Staudt db, $P=3.00 \times 10^{-8}$; Figure 5C), while there was upregulation of STAT3-repressed genes (DAUER_STAT3 TARGETS_DN, MSigDB, $P=9.3 \times 10^{-30}$; Figure 5C). Along these lines, the treatment of OCI-Ly3 and TMD8 cells with $100 \mathrm{nM}$ or $1 \mu \mathrm{M}$ compound 3 for 8 or 24 hours decreased the levels of phosphorylated STAT3 (p-STAT3) (Y705) (Figure 5D), which is a marker of STAT3 activation (41). ABC DLBCLs rely on IL-10 signaling and the JAK/STAT pathway for survival signaling (41, 42). MALT1 may thus be required to maintain the activity of these pathways, suggesting its crosstalk with IL-10 and STAT3 signaling pathways and that this crosstalk may be linked to MALT1 regulation of Roquin, which ultimately modulates IL1O mRNA levels. In contrast, at 24 hours, the OCI-Ly3 compound 3 data set revealed enrichment for the NF- $\kappa \mathrm{B}$ signature in both up- and downregulated NF- $\kappa \mathrm{B}$ target genes (Figure $5 \mathrm{C}$ ). NF- $\kappa \mathrm{B}$ genes induced at 24 hours included the negative regulators of NF- $\kappa \mathrm{B}$ signaling $R E L B$ and NFKB2 and the I $\mathrm{B}$ genes NFKBIA and NFKBIE, as well as numerous cytokines including the T cell-attracting factors $C C R 7$ and CCL22. Yet, among the downregulated NF- $\kappa \mathrm{B}$ targets at 24 hours were the key ABC DLBCL transcription factors NFKB1, $I R F 4$, and $B A T F$, which are required for the survival of these cells. Consistent with our finding that compound 3 induces apoptosis in MALT1-sensitive cell lines (Figure 4F), we found enrichment of positive regulators of apoptosis in the upregulated genes (Positive regulation of apoptosis, GO:0043065, $P=0.00017$; Figure $5 \mathrm{C}$ ) and enrichment of negative regulators in the downregulated genes after compound 3 treatment (Negative regulation of apoptosis GO:0043066, $P=2.42 \times 10^{-7}$; Figure 5C).

Finally, compound 3 strongly upregulated IFN signaling (IFN Signaling, Reactome, $P=1.4 \times 10^{-25}$; IFNA Pathway, Biocarta, $P=$ $3.1 \times 10^{-5}$; Figure $5 \mathrm{C}$ ) after 24 hours of treatment. This is consistent with a previous study, in which increased IFN expression was found in the effector T cells of MALT1-knockin protease-dead mice (39). In line with this, MHC class II and IL-12-mediated signaling genes, which are induced by IFN (43), were also significantly enriched in the upregulated genes at 24 hours $\left(P=4.5 \times 10^{-5}\right.$ and $P=0.5 \times 10^{-3}$, respectively; Figure $5 \mathrm{C}$ ). The increase in MHC class II genes was 
A

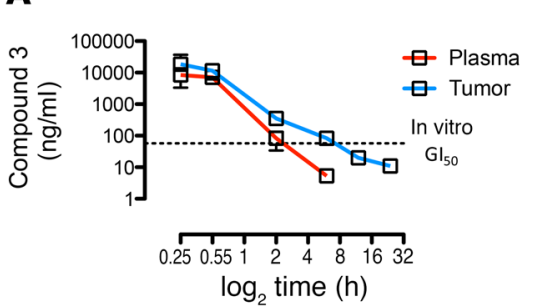

B

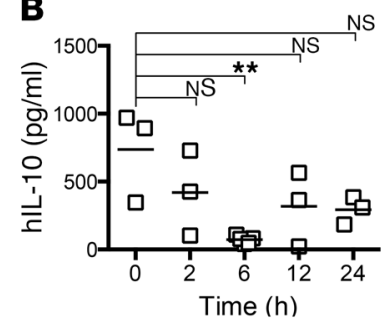

of NF- $\kappa \mathrm{B}$ targets at 8 hours, followed by upregulation at 24 hours, knockdown of MALT1 did not induce an increase in $\mathrm{NF}-\kappa \mathrm{B}$ targets. This might be due to the MALT1 scaffolding activity in compound 3-treated cells that was abolished when MALT1 was absent. Contrary to compound 3 treatment, MALT1 knockdown did not significantly affect Roquin targets or IL-10/ STAT3 target genes (Supplemental Figure $5 \mathrm{~F}$ ).

As shown in Figure 5F, compound 3 inhibition of MALT1 protease activity favors Roquin activity over its target genes, and, as a result, the expression of these genes is decreased. Decreased expression of the Roquin targets IL1O and IL6 in turn causes loss of STAT3 activation. Also, by an unknown mechanism, but potentially through the increase in $\mathrm{NF}-\kappa \mathrm{B}$ signaling observed at 24 hours, compound 3 promotes upregulation of the HLA genes CD74 and CIITA, which are involved in antigen presentation and whose expression is generally low or lost in DLBCL (Figure $5 \mathrm{E})$. These findings suggest that MALT1 inhibition could potentiate the antitumoral a delayed effect, since increased expression was exclusive of the 24-hour time point (Figure 5E).

RNA-seq performed in OCI-Ly3 and TMD8 cells after MALT1 knockdown with 2 independent shRNAs versus a nontargeting control yielded an effect on gene expression similar to that of compound 3. In the case of OCI-Ly3 cells, the normalized enrichment score (NES) was 2.2 and the FDR was less than 0.001 for compound 3-upregulated genes, and the NES was -2.12 and the FDR was less than 0.001 for downregulated genes in OCI-Ly3 shMALT1-knockdown cells. In TMD8 cells, the NES was 1.22 and the FDR was 0.208 for upregulated genes, and the NES was 1.17 and the FDR was 0.212 for downregulated genes in TMD8 shMALT1-knockdown cells (Supplemental Figure 5E). This is probably due to the milder response to both compound 3 and MALT1 knockdown in TMD8 cells. GSEA analysis of MALT1 knockdown showed a significant increase in the IFN signaling signature in both OCI-Ly3 and TMD8 cell lines, which was similar to that observed at 24 hours with compound 3 treatment, although we did not detect as much of an increase in MHC class II genes. On the other hand, while compound 3 caused a downregulation

immune response, an effect that might further enhance the therapeutic effect of these compounds.

Compound 3 inhibits MALT1 paracaspase activity in vivo. These data underline the potential therapeutic impact of MALT1 inhibitors. Therefore, we tested the pharmacokinetic (PK) and PD MALT1-inhibitory properties of compound 3 in vivo. Compound 3 was unlikely to have good oral bioavailability because of the arginine residue. Therefore, we collected mouse PK data upon i.v. and i.p. injection dosing (Supplemental Figure 6) and observed reasonable plasma concentrations. Next, TMD8-xenografted NODSCID mice were treated with $30 \mathrm{mg} / \mathrm{kg}$ compound 3 by i.p. injection, which is more convenient for long-term treatment than i.v. injection. Groups of 3 mice were sacrificed at 15 and 30 minutes and then 2, 6, 12, and 24 hours after injection, and plasma, serum and tumor samples were collected to evaluate the levels of compound 3 and target engagement. Levels of free compound 3 in plasma and tumor tissues were assessed by liquid chromatography tandem mass spectrometry (LC-MS/MS) (Figure 6A). Compound 3 levels remained above in vitro $\mathrm{IC}_{50}$ values for up to 6 hours in tumors (average compound 3 concentration at 6 hours was $146 \pm 50 \mathrm{nM}$ ). 
A

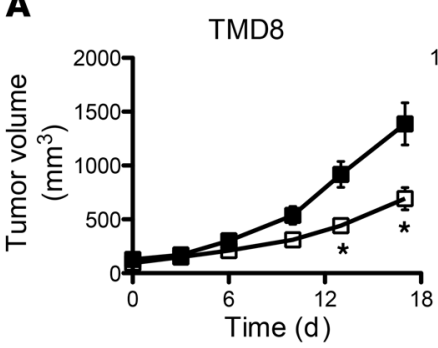

C

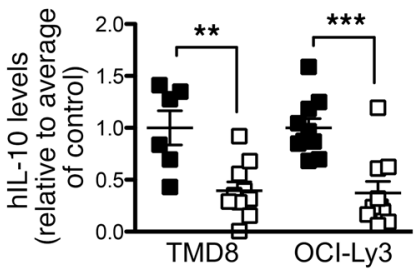

E

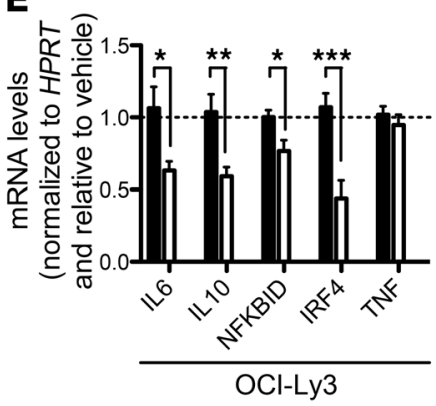

$\mathbf{F}$

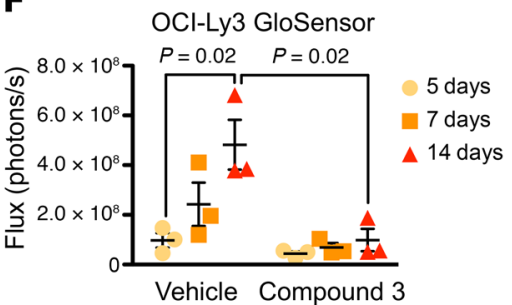

D

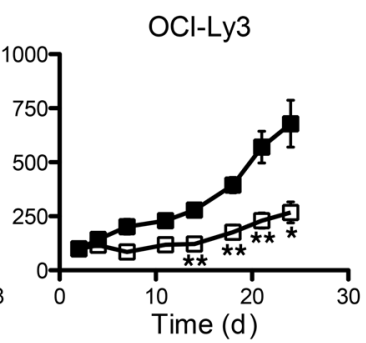

B
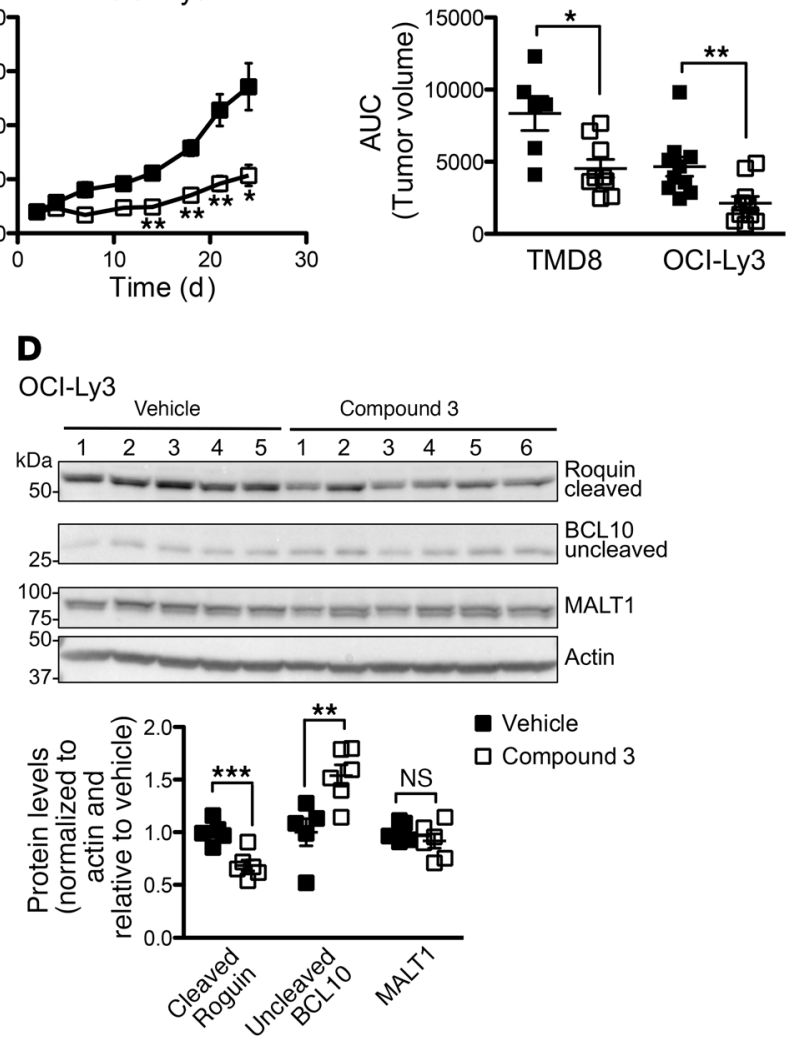

G

OCl-Ly3 GloSensor
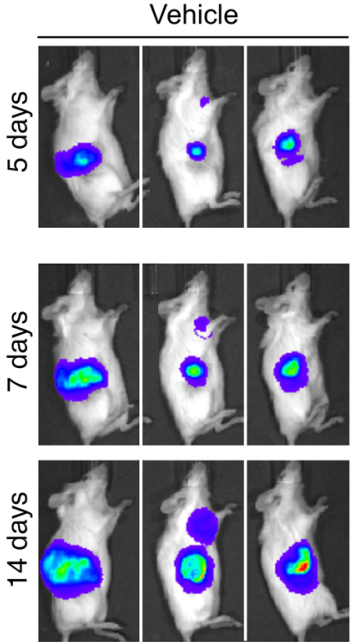

- Vehicle

a Compound 3

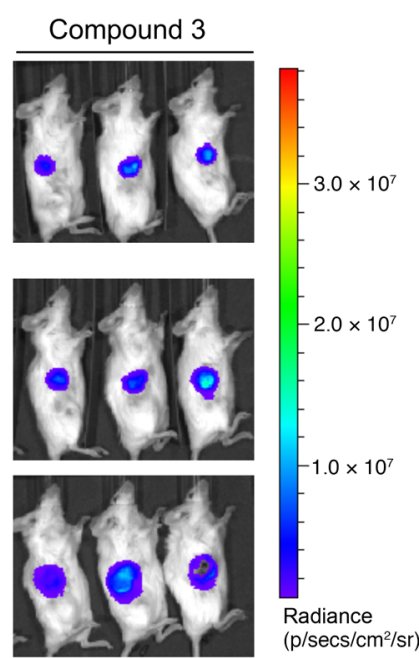

Figure 7. Compound 3 suppresses the growth of ABC DLBCL tumors in vivo. (A) Tumor growth curve for xenografts of the ABC DLBCL cell lines TMD8 (from NOD-SCID mice; $n=9$ /group) and OCI-Ly3 (from NSG mice; $n=10$ /group) following compound 3 treatment. Mice were treated with $30 \mathrm{mg}$ / $\mathrm{kg}$ b.i.d. compound 3 or the same dose of vehicle for 16 or 24 consecutive days, respectively. (B) Tumor volumes for controland compound 3-treated animals bearing TMD8 or OCl-Ly3 xenografts as indicated. The growth of each tumor was measured as the AUC. (C) hIL-10 serum levels at the endpoint of the experiment for TMD8 and OCI-Ly3 xenografts. (D) Western blot results for the MALT1 targets BCL10 and Roquin in OCI-Ly3-xenografted tumors. Graph shows quantification of the indicated genes normalized to actin and relative to vehicle. (E) mRNA levels for compound 3 targets in OCI-Ly3-xenografted tumors ( $n=9$ /group). mRNA levels were normalized to HPRT and are relative to vehicle-treated cells at the indicated time points. (F) Live imaging of MALT1 proteolytic activity in xenografted $\mathrm{OCl}$ Ly3 MALT1-GloSensor cells at the indicated time points. NSG mice were treated b.i.d. with $30 \mathrm{mg}$ / $\mathrm{kg}$ as in A. (G) Bioluminescence signal intensity quantification. Images are of mice used in the study. Data represent the mean \pm SEM. ${ }^{*} P \leq 0.05,{ }^{* *} P \leq 0.01,{ }^{* * *} P$ $<0.001$ (A-E), and $P=0.02$ (F), by unpaired, 2-tailed Student's $t$ test.

We studied target engagement in the same experiment by measuring human IL-10 (hIL-10) levels in collected serum samples. Since previous studies had shown that IL-10 secretion in ABC DLBCL is dependent on MALT1 protease activity $(21,22)$, and given that IL-10 protein has a short half-life (44), we considered hIL-10 in the serum of xenografted mice to an appropriate PD marker of MALT1 inhibition. Indeed, we observed a near-complete suppression of hIL-10 protein expression at 6 hours following compound 3 treatment (Figure 6B). hIL-10 levels increased at later time points, correlating inversely with decreased plasma levels of compound 3 at 12 and 24 hours.
We also explored other markers of response to MALT1 inhibition by Western blot analysis of MALT1-mediated cleavage of BCL10 and Roquin and by qPCR of relevant targets in tumor samples. As shown in Figure 6, C and D, we observed an increase in uncleaved BCL10 protein at 6 hours that was sustained at 12 and 24 hours, although there was certain variability among individuals. In the case of Roquin, its cleavage inhibition was maximal at 2 and 6 hours, and then at 12 and 24 hours, cleaved Roquin returned to baseline levels. Roquin cleavage inhibition preceded hIL-10 protein downregulation (Figure 6B), suggesting that hIL-10 reduc- 
tion could be due to its mRNA degradation by Roquin (17). Indeed, mRNA expression of the Roquin targets IL1O, IL6, NFKBID, IRF4, and $T N F$ also decreased after compound 3 treatment at 6 hours in xenografted tumors as shown by qPCR (Figure 6D). The levels for some of these transcripts remained low at 12 and 24 hours. In the case of $I L 1 O$, mRNA levels were back to control levels at 12 hours, in concordance with hIL-10 protein levels in serum (Figure 6B). IL6 and TNF mRNA levels returned to baseline 24 hours after injection (Figure 6D). Given the PK and PD of compound 3 in mice, we decided to treat mice every 12 hours (b.i.d.) to study the efficacy of compound 3 in vivo.

Compound 3 inhibits the growth of MALT1-dependent $A B C$ $D L B C L$ xenografts. For efficacy studies, we engrafted TMD8 or OCI-Ly3 ABC DLBCL cells and OCI-Ly1 GCB DLBCL cells into the right flank region of NOD-SCID (TMD8 and OCI-Ly1) or NSG mice (OCI-Ly3). Once tumors reached approximately 100 $\mathrm{mm}^{3}$ in volume, the mice were randomized to receive $30 \mathrm{mg} /$ $\mathrm{kg}$ b.i.d. compound 3 or vehicle by i.p. injection ( $n=10$ mice for each treatment of TMD8 and OCI-Ly3; $n=4$ mice for OCI-Ly1). Animals were sacrificed 4 hours after the last injection of the drug or vehicle. Compound 3 suppressed the growth of both TMD8 ( $P=0.025$, 2-tailed Student's $t$ test $)$ and OCI-Ly3 $(P=0.003$, 2-tailed Student's $t$ test) xenografts compared with vehicle (Figure 7, A and B), while it did not affect the growth of OCI-Ly1 xenografts (Supplemental Figure 7, A and B). The body weight of animals was not affected by compound 3 treatment, and it was similar to that of vehicle-treated mice (Supplemental Figure 7C). Histological examination using a TUNEL assay showed a significant increase in apoptotic cells in compound 3-treated TMD8 $(P=0.0045$, 2-tailed Student's $t$ test) and OCI-Ly3 ( $P=0.0002$, 2-tailed Student's $t$ test) xenografts relative to their respective vehicle controls (Supplemental Figure 7D). Representative TUNEL images are shown in Supplemental Figure 7E.

To evaluate MALT1 suppression by compound 3 in treated tumors, we examined hIL-10 levels in serum. As shown in Figure 7C, compound 3 treatment resulted in a significant decrease in serum levels of hIL-10 in TMD8 ( $P=0.003$, 2-tailed Student's $t$ test) and OCI-Ly3 ( $P=0.0003$, 2-tailed Student's $t$ test) xenografted mice. OCI-Ly1 xenografts did not express any detectable levels of hIL-10. We also evaluated the effect of compound 3 on BCL10 and Roquin cleavage by MALT1 in responder xenografts. As shown in Figure 7D for OCI-Ly3 and Supplemental Figure 7F for TMD8, compound 3 treatment significantly decreased the levels of cleaved Roquin in OCI-Ly3 $(P=0.002)$ but not in TMD8 xenografts, in which the reduction was not significant. However increased levels of uncleaved BCL10 were significant in both cell lines $(P=0.009$ and $P=0.002$, 2-tailed Student's $t$ test, for OCI-Ly3 and TMD8, respectively), reflecting the suppression of MALT1 protease activity. Next, we tested the levels of Roquin targets at the mRNA level, and, as shown in Figure 7E and Supplemental Figure 7G, compound 3 treatment resulted in a significant decrease in the levels of IL6,IL1O, NFKBID, and IRF4 in OCI-Ly3 xenografts $(P=0.03 ; P=0.005 ; P=0.02$ and; $P=$ $9.8 \times 10^{-4}$ respectively, 2-tailed Student's $t$ test). We observed a greater individual variability in TMD8 xenografts, in which only NFKBID mRNA levels were significantly decreased $(P=$ 0.01, 2-tailed Student's $t$ test). Therefore, compound 3 target engagement was verified in vivo in the blood and tumors of xenografted mice.

We next generated OCI-Ly3-GloSensor reporter xenografts to quantify the degree of MALT1 suppression in vivo during treatment. MALT1 activity was evaluated at 5, 7, and 14 days of $30 \mathrm{mg} /$ $\mathrm{kg}$ b.i.d. compound 3 treatment. A significant decrease in bioluminescence was observed in animals treated with compound 3 compared with vehicle on day 14 ( $P=0.02$, 2-tailed Student's $t$ test) (Figure $7 F$ ), indicating a sustained suppression of MALT1 in vivo by compound 3. Representative bioluminescence images of xenografted mice are shown in Figure 7G.

Ex vivo-cultured DLBCL patient-derived xenografts maintain MALT1 dependency and respond to compound 3. Finally, to determine the effect of compound 3 on the growth and survival of primary human DLBCL, we cultured DLBCL patient-derived xenograft (PDX) samples ex vivo. We collected tumors from mice xenografted with PDX samples for maintenance and expansion. Tumor masses were digested as indicated in Methods, and DLBCL single cells were isolated. Cells were cultured in the presence or absence of the engineered murine cell line 40LB as previously reported for mouse splenocytes (45), and in either 2D or 3D conditions, depending on their need. Samples were grown with the minimal requirements allowing their proliferation, which was measured as CFSE dilution. We obtained 7 PDX samples, 6 DLBCL samples (3 GCB-like and 3 non-GCB-like according to IHC classification), and 1 follicular lymphoma (FCL) sample. Protein extracts from all samples were kept to determine MALT1 activation status in the primary samples. As expected, GCB DLBCL and FCL samples showed no cleavage bands for Roquin or CYLD and had high levels of uncleaved BCL10 (Figure 8A). Conversely, the 3 non-GCB DLBCL presented cleavage bands for Roquin and CYLD and low levels of uncleaved BCL10 compared with the rest of the samples (Figure 8A), indicating that MALT1 is active in these specimens in ex vivo culture conditions. Next, we sought to characterize the response to compound 3 in these specimens. Cells were CFSE labeled and treated every 3 days for a total of 6 days under the specified conditions. The following criteria were established to assign a sample as being evaluable: (a) vehicle control cell viability of greater than $50 \%$ at the end of the experiment on day 6 and no lower than the starting percentage of viability; and (b) CFSE dilution in the vehicle control sample showed active proliferation in culture. Four specimens were evaluable for growth inhibition and survival under those conditions. As shown in Figure 8B, cell counts significantly decreased in response to compound 3 in a dose-dependent manner in the non-GCB DLBCL specimens, which had active MALT1. CFSE dilution was dose dependent in non-GCB specimens DLBCL-5 and DLBCL-6 (Figure 8C). DLBCL 6 showed the greater effect in proliferation inhibition. Prompted by our finding that the CARD11-mutant cell line OCI-Ly3 was the cell line most sensitive to MALT1 inhibition, we assessed the mutational status of CARD11 in all the PDX specimens tested. Again, the specimen that was most sensitive to MALT1 inhibition, DLBCL 6, carried a CARD11 F130I mutation, which has been previously reported to be a CARD11activating mutation in ABC DLBCL (6). Mutations in CARD11 directly activate the CBM complex and MALT1 protease activity and could make tumors especially dependent on MALT1 activity. 
A
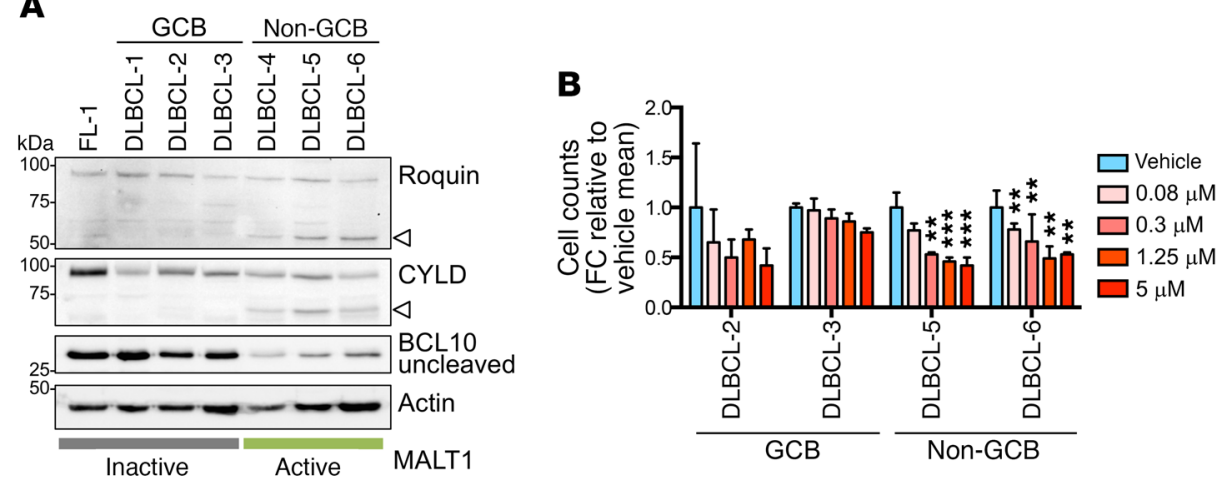

C

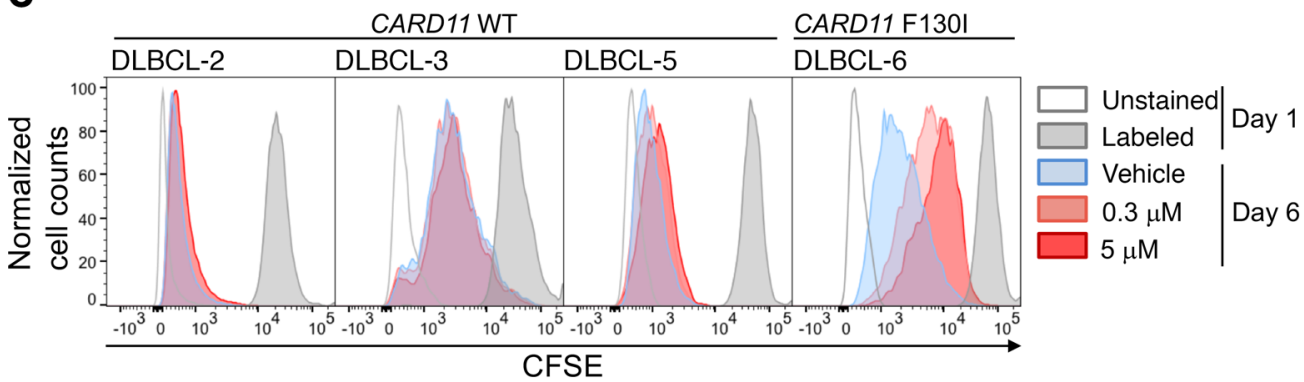

D

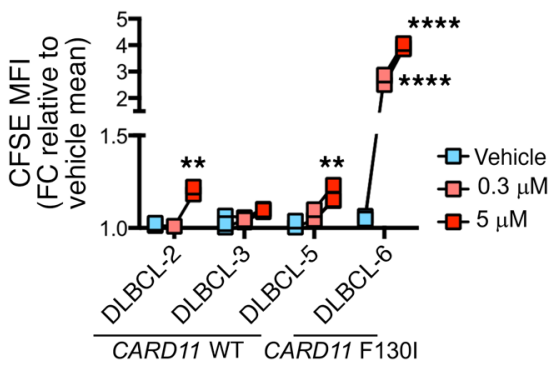

\section{E}

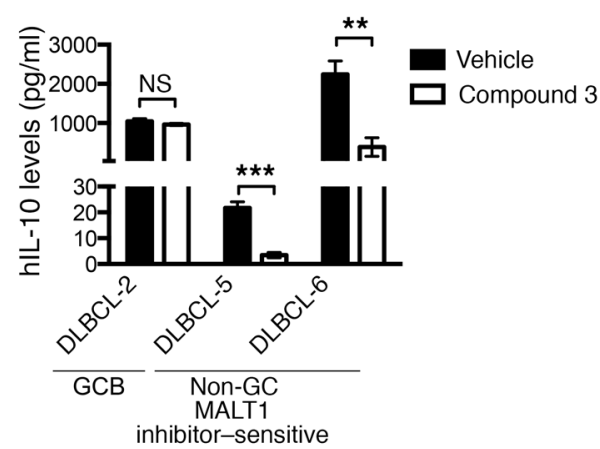

Figure 8. Compound 3 suppresses the growth of DLBCL PDXs ex vivo. (A) Western blots for MALT1 targets Roquin, CYLD, and uncleaved BCL10 in PDX specimens were used to establish the MALT1 activation state in each specimen. (B) Cell counts relative to vehicle-treated cells for the indicated DLBCL PDX specimens ex vivo. Four different concentrations of compound 3 were assayed. Data represent the mean \pm SD of 1 representative experiment. Each experiment was performed at least twice with similar results. (C) CFSE dilution assay results for 2 different concentrations of compound 3 in the indicated PDX specimens. (D) CFSE MFI FC relative to the mean of the vehicle-treated cells. (E) hIL-10 levels in the supernatant of PDX ex vivo culture. Data represent the mean \pm SEM of 1 representative experiment performed in quadruplicate. ${ }^{* *} P$ $\leq 0.01,{ }^{* *} P<0.001$, and ${ }^{* * *} P<$ 0.0001 , by ANOVA with Dunnett's multiple comparisons adjustment (B and $\mathbf{D}$ ) and unpaired, 2-tailed Student's $t$ test (E).
We next measured IL-10 secretion by PDXs on day 6. IL-10 secretion inhibition by compound 3 was restricted to non-GCB primary PDX samples (Figure 8D). IL-10 levels were decreased by compound 3 in DLBCL-5 and DLBCL-6, while the compound did not affect DLBCL-2 IL-10 levels. DLBCL-3 did not express any IL-10.

Overall, in vivo xenograft and ex vivo primary data indicate that MALT1 active site therapeutic targeting using substrate-mimetic peptides is effective at suppressing the growth of ABC DLBCL.

\section{Discussion}

MALT1 is required for the survival of ABC DLBCLs, other types of lymphomas such as mantle cell lymphoma (46), primary effusion lymphoma (47), and chronic Lymphocytic leukemia (48), and potentially other tumors, hence, there is great interest in developing potent inhibitors for this target. MALT1 is part of the CBM complex, which is central to BCR activation. MALT1 functions as a scaffold to activate NF- $\kappa \mathrm{B}$ and as a protease to modulate the abundance of at least 9 protein targets, including itself (49). Accordingly, inhibition of its protease activity is sufficient to inhibit the growth of subsets of ABC DLBCL and MCL cell lines and primary specimens $(23,46)$. Although tool compounds that target MALT1 were previously reported, none of them was suitable for clinical testing for various reasons (23-25).

Here, we have developed a new class of irreversible MALT1 inhibitors inspired by the Z-VRPR-fmk tetrapeptide and based on the structures of known MALT1 natural substrates, positional scanning libraries $(31,32)$, and MALT1 co-crystal structures with Z-VRPR-fmk $(31,33)$. Several rounds of SARs prompted us to lead compound 3, an improved substrate-mimetic peptidic inhibitor of MALT1 protease activity. Compound 3 binds irreversibly to the active site cysteine of MALT1. Covalent catalysis is characteristic of all cysteine proteases; substrates react covalently to the active site cysteine and form a substrate-enzyme intermediate. The activity of compound 3 as a MALT1 inhibitor is absolutely dependent on its covalent binding to MALT1 C464, since a reversible version of compound 1 is inactive. While other substrate-mimetic compounds promoted the formation of stable, dimeric species of MALT1, as previously shown for Z-VRPR-fmk (31), compound 3 induced MALT1 oligomerization in vitro. Whether this mechanism contributes to the increased potency of compound 3 in cells 
has not been elucidated. However, it is conceivable that such mechanism could affect normal CBM complex dynamics.

One of the challenges in the development of MALT1 inhibitors has been the difficulty of testing MALT1 activity in cells with reliability and high-throughput techniques. Most studies have relied on low-throughput assays based on Western blotting or indirect assays based on differential killing of MALT1-dependent and -independent cell lines. Here, we report a high-throughput MALT1 protease activity in-cell reporter system. Our activation-based assay to study MALT1 inhibition in Raji cells provides a robust high-throughput assay to evaluate MALT1 inhibition. Moreover, we developed an OCI-Ly3 MALT1-GloSensor cell line to study in vivo target engagement that allows longitudinal studies of in vivo target engagement and drug PD.

In addition, using compound 3, we studied the consequences of MALT1 protease inhibition at the transcriptional level. MALT1 inhibition triggered gene downregulation at 8 hours (early effects) that was followed by gene upregulation at 24 hours (late effects). Down-regulated pathways by MALT1 inhibition included NF- $\kappa$, IL-10, and JAK-STAT signatures, among others. Remarkably, but not unexpectedly, a Roquin signature ("Roquin targets") was among the most significantly negatively enriched signatures. In fact, Roquin targets NFKBID, IL1O, and IL6 were among the most downregulated genes by compound 3. Notably, Nfkbid-knockout animals show a phenotype very similar to that of MALT1-knockin protease-dead animals (50-53). Indeed, both mouse strains show lack of marginal zone and peritoneal B-1 B cells and impaired lymphocyte activation and germinal center formation (54). Both also lack mature Tregs and show resistance to Th17-dependent autoimmune encephalomyelitis (EAE) $(50,55)$.

Compound 3 downregulated IL-10 protein expression in vitro and in vivo, and, in concordance, IL-10- and JAK-STAT-related transcriptional signatures were downregulated after compound 3 treatment. MALT1 inhibition by compound 3 also led to decreased STAT3 phosphorylation in ABC DLBCL cells. Of note, IL-10 signaling through IL-10RA and its signaling pathway (JAK/STAT3) was previously shown to be critical for ABC DLBCL survival (41). It is thus possible that disruption of IL-10 signaling contributes to the antilymphoma effect of MALT1 inhibitors. IL-10 constitutes, therefore, a candidate biomarker to test target engagement in patients treated with MALT1 inhibitors.

Among upregulated pathways by compound 3 treatment, the type I IFN pathway stands out as the most prominently enriched. Type I IFNs play an essential part in natural cancer immune surveillance, mediating effects on tumoral cells (limiting proliferation and promoting senescence and cell death) and the immune system (activation of innate immunity) $(56,57)$. Therefore, upregulation of type I IFNs by MALT1 inhibition (along with MHC class II and IL-12 signaling upregulation) could be exploited therapeutically to improve the response to immune checkpoint drugs (e.g., anti-PD-1 and anti-CTL4 antibodies). However, the timing of the treatments should be carefully planned and assessed in order to avoid unwanted effects (e.g., autoimmune responses).

In summary, compound 3 is a substrate-mimetic peptidic covalent MALT1 inhibitor and is quite selective in vitro and in vivo. Compound 3 has allowed us to study the downstream effects of MALT1 inhibition at the transcriptional level and to uncover
MALT1 protease-related effects on JAK/STAT and type I IFN signaling. Compound 3 was efficacious in vivo against ABC DLBCL xenografted tumors when used b.i.d. and provides the basis for the development of clinically tractable MALT1 protease inhibitors for use in patients with B cell lymphomas and other tumors.

\section{Methods}

Further information can be found in Supplemental Methods.

Compound synthesis. The procedures for the preparation of compounds 2, 3, and 4 are described in the international patent application WO2017040304 (58). Compound 1 and its reversible control were prepared by very similar methods.

Cell culture. OCI-Ly1, OCI-Ly3, and OCI-Ly10 were cultured in Iscove's medium supplemented with $20 \%$ FBS. The remaining cell lines were cultured in RPMI supplemented with $10 \%$ FBS and 10 mM HEPES. HBL-1, HLY-1, and U2932 were obtained from Jose A. Martinez-Climent (Universidad de Navarra, Pamplona, Spain); OCI-Ly3 cells were obtained from Anas Younes (Memorial Sloan Kettering Cancer Center, New York, New York, USA); OCI-Ly1, OCILy7, and OCI-Ly10 cell lines were obtained from the Ontario Cancer Institute (OCI); TMD8 was obtained from Louis M. Staudt (National Cancer Institute, Bethesda, Maryland, USA); RC-K8 and SU-DHL-4 were obtained from the Deutsche Sammlung von Mikroorganismen und Zellkulturen or German Collection of Microorganisms and Cell Cultures GmbH (DSMZ); and SU-DHL-2 was obtained from American Type Culture Collection (ATCC). All cell lines were authenticated by the University of Arizona Genetic Core and grown in the presence of penicillin $\mathrm{G}$ and streptomycin and $2 \mathrm{mM} \mathrm{L}$-glutamine at $37^{\circ} \mathrm{C}$ in a humidified atmosphere of $5 \% \mathrm{CO}_{2}$.

GloSensor assay. The pGloSensor plasmid was custom designed to have 2 luciferase domains linked by the MALT1 proteolytic substrate sequence of RelB, MALT1-GloSensor reporter (Promega). Cleavage of the linker sequence by MALT1 allows conformational changes promoting large increase in luciferase activity. The assay was validated in 293T cells and subsequently optimized for 384-well plates. Raji-GloSensor cells were generated by electroporation (Amaxa) of pGloSensor containing the RelB cleavage sequence, followed by antibiotic selection. For further validation, Raji MALT1-GloSensor cells were transfected by electroporation (Amaxa) with 2 shRNAs targeting MALT1 and 1 nontargeting hairpin. MALT1-GloSensor was then subcloned into the lentiviral backbone pLEX_306, which was a gift from David Root (Broad Institute of MIT and Harvard, Cambridge, Massachusetts, USA) (Addgene plasmid 41391) and then used to infect OCILy3. Following antibiotic selection, a single clone was isolated by limiting dilution, authenticated by short tandem repeat (STR) profiling and then used for in vivo experiments (OCI-Ly3 MALT1-GloSensor).

MALT1-compound complex crystallization and structure determination. Human MALT1 (UniProtKB: Q9UDY8) 339-719 was overexpressed and purified as described previously (31). The excess amount of compound was incubated with MALT1 for 2 to 16 hours, followed by Superdex 200 (GE Healthcare Life Sciences) purification. The compound 2-MALT1 dimer was crystallized in $0.1 \mathrm{M}$ 2-( $\mathrm{N}$-morpholino) ethanesulfonic acid (MES), pH 6.0, $0.2 \mathrm{M} \mathrm{CaAC,} 2 \%$ benzamidine, and $10 \%$ isopropanol. The compound 3-MALT1 oligomer was treated with 1:200 chymotrypsin (Hampton Research) for 2 hours and run through a Superdex 200. The tetramer peak was concentrated to 2 $\mathrm{mg} / \mathrm{ml}$ and crystallized in $0.1 \mathrm{M} \mathrm{NaAc}, \mathrm{pH} 4.6,0.4 \mathrm{M} \mathrm{NH}_{4}$ tartrate, 
5\% 1,3-butanediol, and 0.3 M NDSB-256. Structures were solved by molecular replacement using previous MALT1 structures (Protein Data Bank [PDB] codes 3UOA and 3V4O). Phenix, CCP4, and Coot were used in model building and refinement (59-61). PyMOL was used for molecular visualization and structure display (PyMOL Molecular Graphics System, version 2.0, Schrödinger LLC).

RNA-seq. RNA was extracted as described above. RNA concentration was determined using Qubit (Life Technologies, Thermo Fisher Scientific), and integrity was verified using the Agilent 2100 Bioanalyzer (Agilent Technologies). Libraries were generated using the TruSeq RNA Sample Kit (Illumina). First-strand synthesis was performed using random oligonucleotides and Superscript III (Invitrogen, Thermo Fisher Scientific). After second-strand synthesis, a 200-bp paired-end library was prepared following the Illumina paired-end library preparation protocol. Paired-end sequencing (PE50) was performed on the Illumina HiSeq 2000. RNA-seq results were aligned to hg19 using STAR (62) and annotated to RefSeq using the Rsubread package (Bioconductor) (63). The RNAseq data were deposited in the NCBI's Gene Expression Omnibus (GEO) database (GEO GSE108293).

Pathway analysis. Unsupervised pathway analysis (parametric assessment of gene set enrichment [PAGE]) was performed using information theoretic pathway tool as described in Goodarzi, et al. (64) Briefly, informative pathways of nonoverlapping gene groups were identified. Pathway annotations were obtained from gene signatures from the Staudt Lab Signature database (65), MSigDB C2 (66) (http:// www.broadinstitute.org/msigdb) and Biological Process annotations of the Gene Ontology (GO) database (http://www.geneontology.org). Only human-curated annotations with 5 or more or 300 or fewer genes from the GO database were evaluated. This pathway analysis tool estimates how informative each pathway is regarding target gene groups and applies a randomization-based statistical test to assess their significance. The significance threshold was set at a $P$ value of less than 0.005. We estimated the FDR by randomizing the input profiles iteratively on shuffled profiles using identical parameters and thresholds. The FDR was always less than $5 \%$. For each informative pathway, we used hypergeometric distribution, as described in Elemento et al. (67), to determine the extent of pathway overrepresentation in the target gene group.

GSEA. Analysis of gene set enrichment was performed using the GSEA algorithm as described in Subramanian, et al. (68). Enrichment was assessed using the GSEA algorithm against preranked gene lists of $\log _{2}$ ratio expression in compound 3 versus vehicletreated samples in all cases.

$P K$. Plasma and tissue samples were mixed with acetonitrile (1:5 $\mathrm{v} / \mathrm{v}$ or $1: 5 \mathrm{w} / \mathrm{v}$, respectively) to precipitate protein and extract the compound from tissue lipids. Tissue samples were disrupted with a probe tip sonicator. Samples were then centrifuged at $16,000 \mathrm{~g}$, and the drug level in the supernatant was determined by LC/MS-MS. LC was performed on an UFLC XR instrument (Shimadzu) and MS on an API5500 instrument (AB Sciex). The peak area was compared, with standard curves prepared in blank plasma and tissue matrix from vehicle-treated animals. Tissue concentrations were calculated as nanograms of compound per milligram of tissue and converted to the concentration assuming a tissue density of $1 \mathrm{~g} / \mathrm{ml}$.

Mouse xenograft experiments. Eight- to ten-week-old female mice were purchased from The Jackson Laboratory and housed in a clean environment. NOD.CB17-Prkdc ${ }^{\text {scid }}$ mice were used for TMD8 and
OCI-Ly1 engraftment, while NOD.Cg-rkdc ${ }^{\text {scid }}$ Il2rg ${ }^{\text {tm1Wil }} /$ SzJ (NSG) mice were used for OCI-Ly3 engraftment. Both mouse strains were purchased from The Jackson Laboratory. Mice were injected s.c. with $10^{7}$ low-passage cells for TMD8 or $5 \times 10^{6}$ cells for OCI-Ly3 and OCILy1 suspended in PBS/Matrigel at 1:1. Treatment was initiated when tumors reached an average size of $100 \mathrm{~mm}^{3}$. Compound 3 was prepared every 4 to 6 days in sterile saline solution ( $\mathrm{pH} 7$ ), stored at $4^{\circ} \mathrm{C}$, and administered i.p. every 12 hours. Tumor size was measured with a digital caliper 2-3 times a week. Tumor volumes were calculated using the following formula: smallest diameter ${ }^{2} \times$ largest diameter $\times 0.5$.

Patient-derived tumor xenograft models and patient-derived tumor xenograft cell culture. Established patient-derived tumor xenograft (PDTX) samples were s.c. injected into NSG mice and allowed to grow until the tumor volume reached $1<1,500 \times \mathrm{mm}^{3}$. PDTX lines were previously analyzed and molecularly annotated (Sandra and Edward Meyer Cancer Center PDTX Shared Resource, Weill Cornell Medicine, New York, New York, USA). Once the tumors reached a sufficient volume, the mice were euthanized and the tumors extracted. The tumors were then dissociated into single cells using a phosphate buffer containing collagenase A $(25,000 \mathrm{U} / \mathrm{ml})$, dispase II $(12.5 \mathrm{U} / \mathrm{ml})$, and DNAse $(500 \mathrm{U} / \mathrm{ml})$. Single cells were mixed with irradiated $40 \mathrm{LB}$ cells (30 Gy $\gamma$ irradiation) at a 10:1 ratio of primary DLBCL/4OLB. The cell mixture was then used to fabricate 3D organoids in 96-well plates using methods similar to those described in ref. 45 , with $20 \mu \mathrm{l}$ 3D organoids containing 3\% silicate nanoparticles and a 5\% gelatin-IMDM medium solution. The 3D organoids were cultured in IMDM medium containing $20 \%$ FBS supplemented with antibiotics and normocin (InvivoGen) for 6 days, doubling the volume of medium after 3 days. The cell mixture was exposed to four 1:3 serial dilutions of compound 3 starting at $5 \mu \mathrm{M}$ or to vehicle control (DMSO) in triplicate for 6 days, with treatment a second time on day 3. After 6 days of exposure, cell viability was assessed by flow cytometry using DAPI staining. Supernatant was collected, and hIL-10 was quantified using the hIL-10 ELISA Kit from eBioscience according to the manufacturer's instructions.

Study approval. All procedures involving animals followed NIH protocols and were approved by the IACUC of Weill Cornell Medicine (protocol 2016-0019). Deidentified patients' samples were obtained with informed consent and xenografted under Weill Cornell Medicine's IRB-approved protocol 1410015560A002 and IACUCapproved protocol 2014-0024.

Statistics. All statistical analyses were performed with GraphPad Prism 6 (GraphPad Software). A $P$ values of less than 0.05 was considered statistically significant. An unpaired, 2-tailed Student's $t$ test was used, except when multiple parameters and comparisons required ANOVA with a multiple comparisons test.

\section{Author contributions}

LF, DAS, HW, NSG, AM, and UP designed the research. LF, QQ, JMH, GC, IU, MD, MX, GD, NB, and SC conducted experiments and/or acquired data. LF, QQ, JMH, GC, MT, and DAS analyzed data. GP and GI provided PDX specimens. The manuscript was written by LF, DAS, and AM and edited by QQ, GC, MT, GI, HW, and NSG.

\section{Acknowledgments}

We thank GenScript USA Inc. for assistance with evaluation of our lead compound in a protease panel; the Northeast- 
ern Collaborative Access Team (NE-CAT) at the Advance Photon Source for their assistance with $\mathrm{x}$-ray data collection; the Epigenomics Core Facility at Weill Cornell Medicine for their assistance with RNA-seq; the Flow Cytometry Core Facility at Weill Cornell Medicine for their assistance with cell sorting; and the Drug Metabolism and Pharmacokinetics laboratory at The Scripps Research Institute for their assistance with PK studies. We thank the Sandra and Edward Meyer Cancer Center PDTX Shared Resource for their support for the PDTX studies. We thank Sucharity Mistry for her assistance with the initial draft. AM, NSG, and HW receive funding from Janssen Pharmaceuticals and the NIH (R01 CA182736). AM acknowledges funding from NIH grant RO1 CA187492. AM and GI acknowledge funding from the Leukemia and Lymphoma Society (LLS)
Specialized Center of Research (SCOR) (grant 7012-16). LF acknowledges funding from the US Department of Defense (W81XWH-15-1-0418).

Address correspondence to: Lorena Fontan or Ari M. Melnick, Weill Cornell Medical College, Cornell University, 413 E. 69th Street BB-1462, New York, New York 10021, USA. Phone: 646.962.6729; Email: lof2005@med.cornell.edu (L. Fontan). Phone: 646.962.6726; E-mail: amm2014@med.cornell.edu (A.M. Melnick). Or to: Nathanael S. Gray, Department of Biological Chemistry and Molecular Pharmacology, Department of Cancer Biology, Dana-Farber Cancer Institute, Harvard Medical School, Longwood Center 2209, 450 Brookline Avenue, Boston, MA, 02115, USA. Phone: 617.582.8590; Email: nathanael_gray@dfci.harvard.edu.
1. Swerdlow HS, et al. WHO Classification of Tumours of Haematopoietic Lymphoid Tissues. Lyon, France: World Health Organization, Lyon, France; 2008.

2. Alizadeh AA, et al. Distinct types of diffuse large B-cell lymphoma identified by gene expression profiling. Nature. 2000;403(6769):503-511.

3. Wright G, Tan B, Rosenwald A, Hurt EH, Wiestner A, Staudt LM. A gene expression-based method to diagnose clinically distinct subgroups of diffuse large B cell lymphoma. Proc Natl Acad Sci U S A. 2003;100(17):9991-9996.

4. Ngo VN, et al. A loss-of-function RNA interference screen for molecular targets in cancer. Nature. 2006;441(7089):106-110.

5. Davis RE, et al. Chronic active B-cell-receptor signalling in diffuse large B-cell lymphoma. Nature. 2010;463(7277):88-92.

6. Lenz G, et al. Oncogenic CARD11 mutations in human diffuse large B cell lymphoma. Science. 2008;319(5870):1676-1679.

7. Kato M, et al. Frequent inactivation of A2O in B-cell lymphomas. Nature. 2009;459(7247):712-716.

8. Compagno M, et al. Mutations of multiple genes cause deregulation of NF-kappaB in diffuse large B-cell lymphoma. Nature. 2009;459(7247):717-721.

9. Ngo VN, et al. Oncogenically active MYD88 mutations in human lymphoma. Nature. 2011;470(7332):115-119.

10. Lenz G, et al. Molecular subtypes of diffuse large B-cell lymphoma arise by distinct genetic pathways. Proc Natl Acad Sci U S A. 2008;105(36):13520-13525.

11. Dierlamm J, et al. Gain of chromosome region $18 \mathrm{q} 21$ including the MALT1 gene is associated with the activated B-cell-like gene expression subtype and increased BCL2 gene dosage and protein expression in diffuse large B-cell lymphoma. Haematologica. 2008;93(5):688-696.

12. Coornaert B, et al. $\mathrm{T}$ cell antigen receptor stimulation induces MALT1 paracaspase-mediated cleavage of the NF-kappaB inhibitor A20. Nat Immunol. 2008;9(3):263-271.

13. Rebeaud F, et al. The proteolytic activity of the paracaspase MALT1 is key in T cell activation. Nat Immunol. 2008;9(3):272-281.

14. Staal J, et al. T-cell receptor-induced JNK activation requires proteolytic inactivation of CYLD by MALT1. EMBO J. 2011;30(9):1742-1752.
15. Hailfinger S, et al. Malt1-dependent RelB cleavage promotes canonical NF-kappaB activation in lymphocytes and lymphoma cell lines. Proc Natl Acad Sci U S A. 2011;108(35):14596-14601.

16. Uehata T, et al. Malt1-induced cleavage of regnase- 1 in $\mathrm{CD} 4(+)$ helper $\mathrm{T}$ cells regulates immune activation. Cell. 2013;153(5):1036-1049.

17. Jeltsch KM, et al. Cleavage of roquin and regnase-1 by the paracaspase MALT1 releases their cooperatively repressed targets to promote $\mathrm{T}(\mathrm{H}) 17$ differentiation. Nat Immunol. 2014;15(11):1079-1089.

18. Elton L, Carpentier I, Staal J, Driege Y, Haegman M, Beyaert R. MALT1 cleaves the E3 ubiquitin ligase HOIL- 1 in activated T cells, generating a dominant negative inhibitor of LUBAC-induced

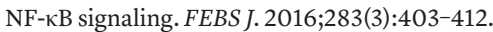

19. Hailfinger S, Schmitt A, Schulze-Osthoff K. The paracaspase MALT1 dampens NF- $\kappa \mathrm{B}$ signalling by cleaving the LUBAC subunit HOIL-1. FEBS J. 2016;283(3):400-402.

20. Ruland J, Duncan GS, Wakeham A, Mak TW. Differential requirement for Malt1 in $\mathrm{T}$ and $\mathrm{B}$ cell antigen receptor signaling. Immunity. 2003;19(5):749-758.

21. Hailfinger S, et al. Essential role of MALT1 protease activity in activated B cell-like diffuse large B-cell lymphoma. Proc Natl Acad Sci U S A. 2009;106(47):19946-19951.

22. Ferch U, et al. Inhibition of MALT1 protease activity is selectively toxic for activated B celllike diffuse large B cell lymphoma cells. J Exp Med. 2009;206(11):2313-2320.

23. Fontan L, et al. MALT1 small molecule inhibitors specifically suppress ABC-DLBCL in vitro and in vivo. Cancer Cell. 2012;22(6):812-824.

24. Nagel D, et al. Pharmacologic inhibition of MALT1 protease by phenothiazines as a therapeutic approach for the treatment of aggressive ABC-DLBCL. Cancer Cell. 2012;22(6):825-837.

25 . Lim SM, et al. Identification of $\beta$-lapachone analogs as novel MALT1 Inhibitors To Treat an aggressive subtype of diffuse large B cell lymphoma. JMed Chem. 2015;58(21):8491-8502.

26. Wood JM, et al. Structure-based design of aliskiren, a novel orally effective renin inhibitor. Biochem. Biophys Res Commun. 2003;308(4):698-705.

27. Kempf DJ, et al. ABT-538 is a potent inhibitor of human immunodeficiency virus protease and has high oral bioavailability in humans. Proc Natl
Acad Sci U S A. 1995;92(7):2484-2488.

28. Kuhn DJ, et al. Potent activity of carfilzomib, a novel, irreversible inhibitor of the ubiquitinproteasome pathway, against preclinical models of multiple myeloma. Blood. 2007;110(9):3281-3290.

29. Vercammen D, et al. Serpin1 of Arabidopsis thaliana is a suicide inhibitor for metacaspase 9. $\mathrm{J} \mathrm{Mol}$ Biol. 2006;364(4):625-636.

30. Hailfinger S, Rebeaud F, Thome M. Adapter and enzymatic functions of proteases in T-cell activation. Immunol Rev. 2009;232(1):334-347.

31. Wiesmann C et al. Structural determinants of MALT1 protease activity. J Mol Biol. 2012;419(1-2):4-21.

32. Hachmann J, et al. Mechanism and specificity of the human paracaspase MALT1. Biochem J. 2012;443(1):287-295.

33. Yu JW, Jeffrey PD, Ha JY, Yang X, Shi Y. Crystal structure of the mucosa-associated lymphoid tissue lymphoma translocation 1 (MALT1) paracaspase region. Proc Natl Acad Sci US A. 2011;108(52):21004-21009.

34. Hachmann J, Salvesen GS. The paracaspase MALT1. Biochimie. 2016;122:324-338.

35. Hachmann J, et al. Probes to monitor activity of the paracaspase MALT1. Chem Biol. 2015;22(1):139-147.

36. Qiao Q, et al. Structural architecture of the CARMA1/Bcl10/MALT1 signalosome: nucleation-induced filamentous assembly. Mol Cell. 2013;51(6):766-779.

37. Wilson WH, et al. Targeting B cell receptor signaling with ibrutinib in diffuse large B cell lymphoma. Nat Med. 2015;21(8):922-926.

38. Yahiaoui A, et al. PI3K $\delta$ inhibitor idelalisib in combination with BTK inhibitor ONO/GS-4059 in diffuse large $\mathrm{B}$ cell lymphoma with acquired resistance to $\mathrm{PI} 3 \mathrm{~K} \delta$ and BTK inhibitors. PLoS One. 2017;12(2):e0171221.

39. Gewies A, et al. Uncoupling Malt1 threshold function from paracaspase activity results in destructive autoimmune inflammation. Cell Rep. 2014;9(4):1292-1305.

40. Leppek K et al. Roquin promotes constitutive mRNA decay via a conserved class of stem-loop recognition motifs. Cell. 2013;153(4):869-881.

41. Béguelin W, et al. IL10 receptor is a novel therapeutic target in DLBCLs. Leukemia. 2015;29(8):1684-1694.

42. Rui L, et al. Epigenetic gene regulation by Janus 


\section{RESEARCH ARTICLE}

kinase 1 in diffuse large B-cell lymphoma. Proc Natl Acad Sci U S A. 2016;113(46):E7260-E7267.

43. Hervas-Stubbs S, et al. Direct effects of type I interferons on cells of the immune system. Clin Cancer Res. 2011;17(9):2619-2627.

44. Mosser DM, Zhang X. Interleukin-10: new perspectives on an old cytokine. Immunol Rev. 2008;226:205-218.

45. Purwada A, et al. Ex vivo engineered immune organoids for controlled germinal center reactions. Biomaterials. 2015;63:24-34.

46. Dai B, et al. B-cell receptor-driven MALT1 activity regulates MYC signaling in mantle cell lymphoma. Blood. 2017;129(3):333-346.

47. Bonsignore L, et al. A role for MALT1 activity in Kaposi's sarcoma-associated herpes virus latency and growth of primary effusion lymphoma. Leukemia. 2017;31(3):614-624.

48. Saba NS, et al. MALT1 Inhibition is efficacious in both naïve and ibrutinib-resistant chronic lymphocytic leukemia. Cancer Res. 2017;77(24):7038-7048.

49. Jaworski M, Thome M. The paracaspase MALT1: biological function and potential for therapeutic inhibition. Cell Mol Life Sci. 2016;73(3):459-473.

50. Kobayashi $S$, et al. The nuclear I $\mathrm{B}$ family protein I $\kappa$ BNS influences the susceptibility to experimental autoimmune encephalomyelitis in a murine model. PLoS One. 2014;9(10):e110838.

51. Pedersen GK, et al. B-1a transitional cells are phenotypically distinct and are lacking in mice deficient in IкBNS. Proc Natl Acad Sci U S A. 2014;111(39):E4119-E4126.

52. Jaworski M, et al. Malt1 protease inactivation efficiently dampens immune responses but causes spontaneous autoimmunity. EMBOJ. 2014;33(23):2765-2781.

53. Yu JW, et al. MALT1 protease activity is required for innate and adaptive immune responses. PLoS One. 2015;10(5):e0127083.

54. Touma M, et al. Impaired B cell development and function in the absence of IkappaBNS.JImmunol. 2011;187(8):3942-3952.

55. Brüstle A, et al. The NF- $\kappa$ B regulator MALT1 determines the encephalitogenic potential of Th17 cells. J Clin Invest. 2012;122(12):4698-4709.

56. Ivashkiv LB, Donlin LT. Regulation of type I interferon responses. Nat Rev Immunol. 2014;14(1):36-49.

57. Snell LM, McGaha TL, Brooks DG. Type I interferon in chronic virus infection and cancer. Trends Immunol. 2017;38(8):542-557.

58. Scott DA, et al, inventors. Cornell University, Dana-Farber Cancer Institute Inc., Children's Medical Center Corp., assignees. Malt1 inhibitors uses thereof. US patent WO2017040304 A1: March 9, 2017.

59. Adams PD, et al. PHENIX: a comprehensive Python-based system for macromolecular structure solution. Acta Crystallogr D Biol Crystallogr.
The Journal of Clinical Investigation

2010;66(Pt 2):213-221.

60. Emsley P, Lohkamp B, Scott WG, Cowtan K. Features and development of Coot. Acta Crystallogr D Biol Crystallogr. 2010;66(Pt 4):486-501.

61. Winn MD, et al. Overview of the CCP4 suite and current developments. Acta Crystallogr D Biol Crystallogr. 2011;67(Pt 4):235-242.

62. Dobin A, et al. STAR: ultrafast universal RNA-seq aligner. Bioinformatics. 2013;29(1):15-21.

63. Liao Y, Smyth GK, Shi W. The Subread aligner: fast, accurate and scalable read mapping by seed-and-vote. Nucleic Acids Res. 2013;41(10):e108.

64. Goodarzi H, Elemento O, Tavazoie S. Revealing global regulatory perturbations across human cancers. Mol Cell. 2009;36(5):900-911.

65. Shaffer AL, et al. A library of gene expression signatures to illuminate normal and pathological lymphoid biology. Immunol Rev. 2006;210:67-85.

66. Liberzon A, et al. Molecular signatures database (MSigDB) 3.0. Bioinformatics. 2011;27(12):1739-1740.

67. Elemento O, Slonim N, Tavazoie S. A universal framework for regulatory element discovery across all genomes and data types. Mol Cell. 2007;28(2):337-350.

68. Subramanian A, et al. Gene set enrichment analysis: a knowledge-based approach for interpreting genome-wide expression profiles. Proc Natl Acad Sci US A. 2005;102(43):15545-15550. 